AUFSATZ

Georg Erber

\title{
China: Land der unbegrenzten Möglichkeiten?
}

\section{China am Scheideweg}

China hat nach seinem rasanten Aufstieg zur zweitgrößten Volkswirtschaft der Welt und seiner damit verbundenen Rolle als eine der großen Handelsnationen ${ }^{1}$ die Phantasie der Politik und der Öffentlichkeit auch für ausländische Unternehmen insbesondere des Westens befeuert. Damit hat China gewissermaßen die USA als Land der unbegrenzten Möglichkeiten abgelöst. Während spätestens im Zuge der globalen Wirtschafts- und Finanzkrise die USA an Strahlkraft als Rollenmodell für wirtschaftliche Entwicklung weltweit verlor, hat China deutlich an Ansehen hinzugewonnen. Als wichtigstes Mitglied der BRICS-Staaten ${ }^{2}$ ist es zu deren Sprecher auch bei den G20-Gipfeln geworden und setzt sich für die Interessen der Entwicklungsländer sowie natürlich der führenden Emerging Market Economies (EME) gegen die Hegemonie des Westens in der Weltwirtschaft ein.

\subsection{Der Renminbi als Weltreservewährung?}

Beim G20-Gipfel in St. Petersburg im Jahr 2013 hat China gemeinsam mit den anderen BRICS-Staaten die Gründung eines eigenen Währungsfonds beschlossen, in den diese Länder einen Betrag von 100 Mrd. US-Dollar einzahlen. ${ }^{3}$ Damit schaffen sich die BRICS-Staaten analog zum ESM (Europäischer Stabilitätsmechanismus - European Stability Mechanism) in Europa einen Reservefonds, um Länder, die in Zahlungsbilanzschwierigkeiten geraten, aus diesem Topf unabhängig vom IWF (Internationaler Währungsfonds) Finanzhilfen gewähren zu können. Das multilaterale System der Kontrolle der Weltwirtschaft unter der Hegemonie der USA mit seinen Institutionen IWF, Weltbank und WHO (Welthandelsorganisation) zerfällt damit zunehmend in eines regionaler Systeme, die zunächst noch als Parallelsysteme zum IWF koexistieren. Auf mittlere Sicht ist die chinesische Regierung bestrebt, den chinesischen Renminbi zu einer der großen Weltreservewährungen zu machen. Dahinter steckt eine Doppelstrategie, einerseits seinen Einfluss beim IWF auszuweiten und gleichzeitig eben einen eigenständigen Währungsreservefonds mit den BRICS-Staaten zu betreiben. China baute zudem in den letzten Jahren seine Goldreserven massiv aus (siehe Tabelle 1). ${ }^{4}$ Während Deutschland, die USA und zahl-

1 Erber 2013 a.

2 Die Abkürzung BRICS steht für die Anfangsbuchstaben der fünf Staaten: Brasilien, Russland, Indien, China und Südafrika.

3 Siehe FAZ 2013 c.

4 Siehe Die Welt 2013 a.

Leviathan, 42. Jg., 1/2014, S. 29 - 666 
reiche andere westliche Länder ihre Goldreserven während des Bretton-Woods-Systems noch zu im Vergleich zu heute einmalig günstigen Goldpreisen von 35 USDollar je Feinunze aufbauen konnte, wird es für die Nachzügler sehr viel teurer.

Tabelle 1: Goldreserven bei den Zentralbanken der Länder mit den größten Goldreserven

\begin{tabular}{|c|l|r|r|r|r|}
\hline Rang & Land & $\begin{array}{c}\text { Goldreser- } \\
\text { ven in Ton- } \\
\text { nen }\end{array}$ & $\begin{array}{c}\text { Marktwert } \\
\text { in Mrd. Euro }\end{array}$ & $\begin{array}{c}\text { Veränderung } \\
\text { auf Sicht von } \\
\text { 4 Jahren }\end{array}$ & $\begin{array}{c}\text { Veräderung } \\
\text { in Prozent }\end{array}$ \\
\hline 1 & USA & $8.133,5$ & 325 & 0,0 & 0,0 \\
2 & Deutschland & $3.391,4$ & 135 & $-21,2$ & $-0,6$ \\
3 & Italien & $2.451,9$ & 98 & 0,0 & 0,0 \\
4 & Frankreich & $2.435,4$ & 97 & $-72,1$ & $-2,9$ \\
5 & China & $1.054,1$ & 42 & 454,1 & 75,7 \\
6 & Schweiz & $1.040,1$ & 41 & $-0,1$ & 0,0 \\
7 & Russland & 957,7 & 38 & 447,8 & 87,8 \\
8 & Japan & 765,2 & 31 & 0,0 & 0,0 \\
9 & Niederlande & 612,5 & 24 & $-1,0$ & $-0,2$ \\
10 & Indien & 557,7 & 22 & 200,0 & 55,9 \\
11 & Portugal & 382,5 & 15 & $-0,1$ & 0,0 \\
\hline \multirow{7}{*}{ Quelle: Bloomberg. } & \multicolumn{4}{c}{ Stand: Februar 2013 } \\
\hline
\end{tabular}

Mittelfristig wird hierdurch das außerordentliche Privileg (exorbitant privilege $e^{5}$ ) der USA mit dem US-Dollar als dominanter Weltreservewährung nachhaltig infrage gestellt. ${ }^{6}$ Bisher können die USA ihre führende Weltreservewährung nutzen, um mittels der US-Notenpresse jedwede Zahlungsbilanzkrise der USA trotz gewaltiger Leistungsbilanzdefizite gegenüber der übrigen Welt und insbesondere hier auch gegenüber China zu begleichen. ${ }^{7}$ China könnte aber zukünftig zumindest entsprechend der Reichweite seiner Renminbi-Zone ${ }^{8}$ (vgl. hierzu auch Abbildung 1) diese Möglichkeiten für sich nutzen und die USA indirekt zwingen, ihre Wirtschaftspolitik in Richtung eines nachhaltigen Leistungsbilanzdefizitabbaus zu korrigieren. Dem

5 Der Begriff des »exorbitant privilege « geht auf Valerie Giscard d'Estaing zurück, der in den 1960er Jahren damit die Möglichkeit der USA bezeichnete, Probleme mit ihrer Zahlungsbilanz durch die US-Notenpresse zu begleichen, während andere Länder zu einer oftmals harten Konsolidierungspolitik gezwungen waren. Am Ende führte diese Haltung der US-Regierung zum Zusammenbruch des Bretton-Woods-Systems, da Frankreich auf die Bezahlung in Gold anstelle von US-Dollar für seine Forderungen an die USA beharrte.

6 Eichengreen 2011.

7 Unvergessen ist der Satz des US-Finanzministers John Connally vom 16. August 1971: »Der Dollar ist unsere Währung, aber euer Problem «; vgl. Volcker, Gyohten 1992, S. 81. Damit wies er alle Wünsche der Europäer nach einer größeren fiskalischen und monetären Disziplin der USA zurück. Letztendlich beschleunigte genau diese Haltung der USA die Entwicklung eines eigenen europäischen Währungsverbunds.

8 China hat bereits zahlreiche bilaterale Freihandelsabkommen geschlossen, darunter eines mit den ASEAN-Staaten und eines mit der Schweiz; vgl. FAZ 2010; FAZ 2013 b. 
Euro als derzeit noch zweitwichtigster Weltreservewährung ${ }^{9}$ erwächst somit jenseits des US-Dollars ein weiterer Konkurrent. Da die zukünftig wachstumsstarken Regionen der Weltwirtschaft nicht in Westeuropa oder den USA liegen werden, dürften sich in den kommenden Jahren und Jahrzehnten die Gewichte weiter zugunsten der BRICS-Länder verschieben. Daran würden die jeweiligen bilateralen Freihandelsabkommen zwischen den Vertretern der Triade, also den USA, Japan und der EU, im Kern nichts ändern können, sollten sie denn demnächst zustande kommen. Chinas ungebremster Rohstoffhunger zieht alle Länder, die ihnen Rohstoffe liefern, in seine wirtschaftliche und politische Abhängigkeit. Das dürfte bereits dann relevant werden, wenn sich die aktuellen Zahlungsbilanzkrisen in Indien und in Brasilien weiter verschärfen. Wird dann der IWF oder der jetzt gegründete Beistandsfonds der BRICS-Staaten für Finanzhilfen in Anspruch genommen werden?

\section{Abbildung 1: China-ASEAN-Freihandelszone}

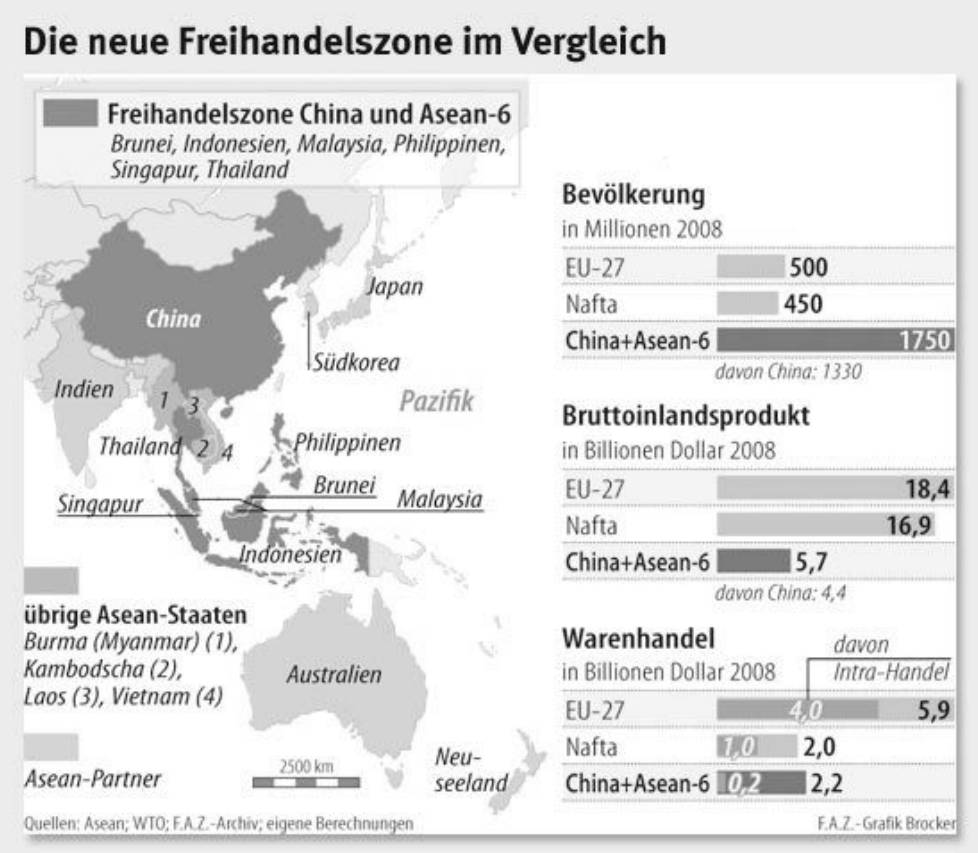

\subsection{China als neue Hegemonialmacht}

China strebt also nicht nur nach internationaler Anerkennung, sondern sieht sich in der Rolle als zukünftige globale Hegemonialmacht sowohl in wirtschaftlicher wie

9 Siehe FAZ 2013 a.

Leviathan, 42. Jg., 1/2014 
auch in politischer Hinsicht. ${ }^{10}$ Zur Ausweitung seiner kulturellen Soft Power ${ }^{11}$ wurden in den zurückliegenden Jahren weltweit zahlreiche Konfuzius-Institute ${ }^{12}$ eingerichtet. Damit kehrt man aus chinesischer Sicht nur wieder zu dem historischen Normalzustand des Reichs der Mitte zurück, den China seinem Selbstverständnis nach bis zum Beginn des Kolonialzeitalters für weite Teile Asiens eingenommen hat. ${ }^{13}$ Die großchinesische Perspektive, die neben Hong Kong und Macao auch Taiwan einschließt, umfasst ein weltweites Netzwerk von Chinesischstämmigen (»Expats «), die in Südostasien (Singapur, Thailand, Myanmar, Malaysia und Indonesien) große Teile der dortigen Wirtschaft dominieren und sich einen wesentlichen Einfluss auf die Wirtschaftspolitik und die Politik dieser Länder sichern. Dieser Einfluss dürfte sich mit der Eröffnung einer Freihandelszone mit den ASEAN-Staaten weiter erhöhen. Zugleich wachsen damit die Spannungen mit Japan, das ebenfalls in diesen Ländern in der Tradition der Great East Asia Co-Prosperity Sphere seine wirtschaftliche Stellung in den zurückliegenden Jahrzehnten erheblich ausgebaut hat.

Daraus erklärt sich eine große Zahl von Territorialkonflikten über See- und Fischereirechte im Chinesischen Meer ${ }^{14}$ (siehe Abbildung 2) und die wachsende Rivalität zwischen China und Japan um den jeweiligen wirtschaftlichen Einfluss in dieser Region. Im Kern beansprucht China im Chinesischen Meer quasi einen Cordon Sanitaire, der es vor ausländischen Bedrohungen vom Meer her schützen soll. Da diese Forderungen über die bisher international üblichen 200-Seemeilenzonen

10 Pettis 2013.

11 »Soft Power (im deutschen auch als weiche Macht bezeichnet) ist ein von Joseph Nye geprägter politikwissenschaftlicher Begriff, der die politische Machtausübung (insbesondere die Einflussnahme in den internationalen Beziehungen) auf Grundlage kultureller Attraktivität, der Ideologie und auch mithilfe internationaler Institutionen beschreibt. Zentrales Merkmal der Soft Power ist die Machtausübung durch die Beeinflussung der Ziele politischer Akteure, ohne dass dazu (wirtschaftliche) Anreize oder (militärische) Bedrohungen eingesetzt werden «; siehe http://de.wikipedia.org/wiki/Soft_Power (Zugriff vom 18.12.2013). Vgl. hierzu Nye 1990, S. 153-171.

12 »Die Konfuzius-Institute sind Institute für Kultur und Sprache, die vom Büro für chinesische Sprachausbildung (Hanban) weltweit gemeinsam mit lokalen Partnern betrieben werden. Sie haben die Aufgabe, Kenntnisse über die chinesische Kultur und Sprache in der Welt zu verbreiten. Vorbild sind vergleichbare Institute für Sprache und Kultur, wie das deutsche Goethe-Institut, das spanische Instituto Cervantes, das British Council oder das portugiesische Instituto Camões. Wegen möglicher Einflussnahme sehen sich diese Institute immer wieder Kritik ausgesetzt «; siehe http://de.wikipedia.org/wiki/KonfuziusInstitut (Zugriff vom 18.12.2013); www.konfuzius-institute.de/

(Zugriff vom 18.12.2013).

13 Pommeranz 2000.

14 Mit dem Begriff Chinesisches Meer fasst man das große Randmeer des Pazifiks zusammen, der das Gelbe Meer (mit seinen nördlichen Ausläufern Golf von Bohai und Koreabucht), das Ostchinesische Meer und das Südchinesische Meer vereinigt. Das Meer liegt zwischen Borneo, Hinterindien, den japanischen Kyūshū-Inseln, der malaiischen Halbinsel, Nordkorea, Südkorea und der Ostküste der Volksrepublik China. 
Abbildung 2: Territorialkonflikte in Ost-und Südostasien, in die China involviert ist

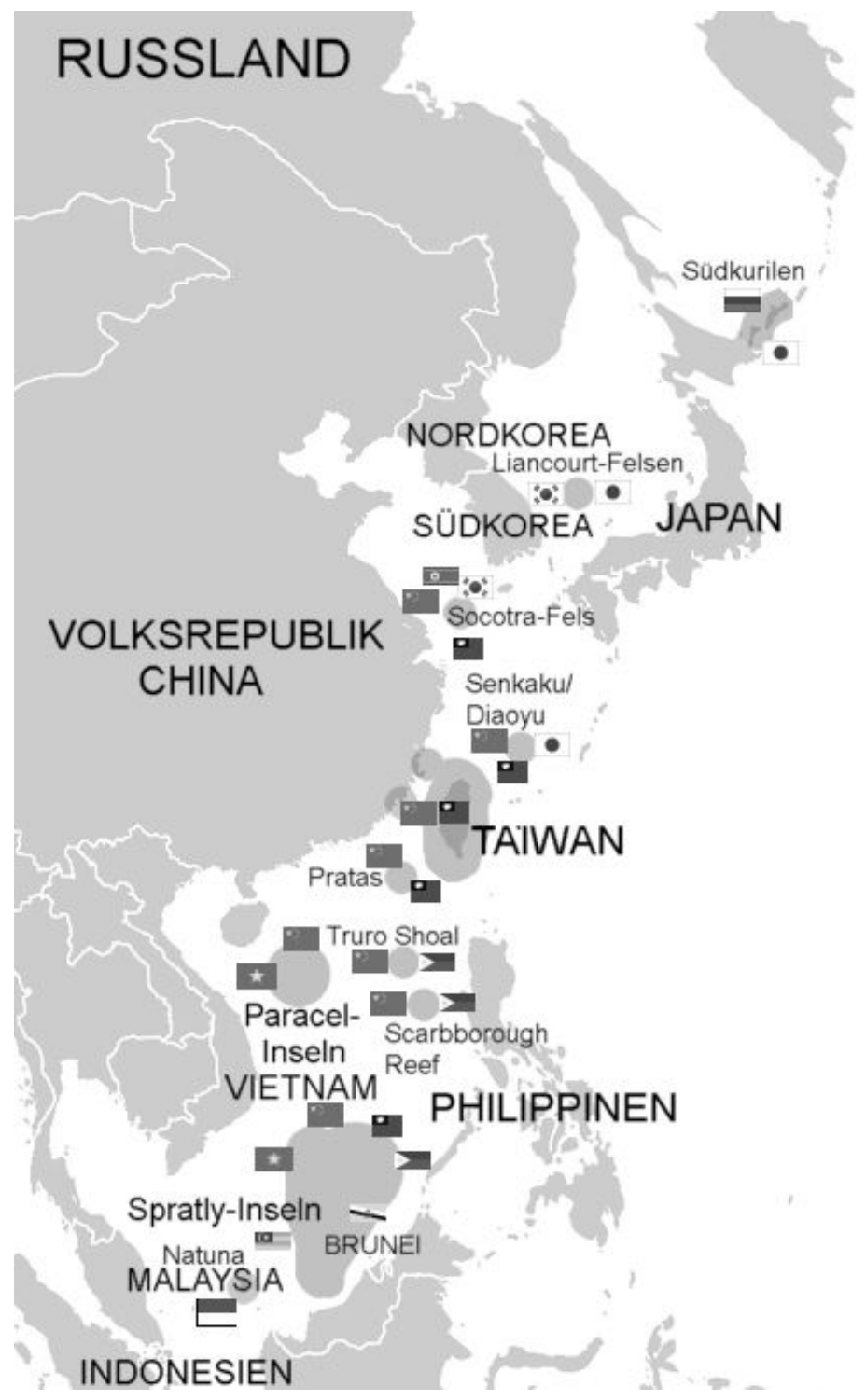

Quelle: Wikipedia Commons.

hinausgehen und zugleich große Teile der Inseln von anderen Anrainerstaaten mitbeansprucht werden, ist hier ein schwelender Konflikt mit China und seinen Anrai- 
nerstaaten angelegt. ${ }^{15}$ Andere Territorialkonflikte bestehen insbesondere auch in der Himalaya-Region mit Indien und Pakistan in der Kashmir-Region sowie in Sikkim mit Indien. Dagegen konnten die Grenzstreitigkeiten zwischen Russland und China am Ussuri, die in den 1960er Jahren zu kriegerischen Auseinandersetzungen geführt hatten, im Jahr 2008 endgültig beigelegt werden. ${ }^{16}$ China hat sich mit der Shanghai Organisation für Zusammenarbeit (SOC) ${ }^{17}$ eine Organisation geschaffen, in der es gemeinsam mit Russland im zentralasiatischen Raum die wirtschaftliche Zusammenarbeit vorantreibt und den Handel mit Russland ausweitet. Man ist insbesondere bemüht, die politischen Spannungen zwischen China und Russland systematisch abzubauen. Damit soll der Einfluss der USA in Zentralasien, der seit dem Zusammenbruch der Sowjetunion zugenommen hatte, wieder eingedämmt werden. Der Rückzug der USA und ihrer Verbündeten aus Afghanistan ist ein erster Meilenstein in dieser Entwicklung.

Der Machtanspruch dürfte heute bereits weit über den historischen Einfluss Chinas in Asien hinausreichen. ${ }^{18}$ China ist zum größten Geberland und Handelspartner in Afrika aufgestiegen und zu einem der wichtigsten Handelspartner für Lateinamerika ${ }^{19}$ und für Australien ${ }^{20}$ avanciert. Mithin sind wirtschaftliche und politische Spannungen bis hin zu militärischen Konflikten beim zukünftigen Aufstieg Chinas

15 Die chinesische Parteiführung pflegt daher auch das Andenken an historische Persönlichkeiten wie Koxinga, ein chinesischer General, der für die Ming-Dynastie Formosa von den Portugiesen zurückeroberte, und Zheng He, ein weiterer chinesischer Admiral, der mit einer gewaltigen chinesischen Flotte unter der Ming-Dynastie auf Entdeckungsfahrten ging, die ihn bis nach Indien und Afrika führten. Damit wird die historische Tradition Chinas als Seemacht gepflegt und zugleich die derzeitigen aktuellen maritimen Ambitionen gegenüber der eigenen Bevölkerung legitimiert.

16 Siehe Die Presse 2008.

17 Zur SCO gehören die Volksrepublik China, Russland, Usbekistan, Kasachstan, Kirgisistan und Tadschikistan. Afghanistan, Indien, Pakistan, der Iran und die Mongolei haben dort einen Beobachterstatus. Als Dialogpartner sind Belarus, Sri Lanka und die Türkei am SCO beteiligt. Als Gäste an den Treffen sind des Weiteren Vertreter von ASEAN, der Commonwealth of Independent States (CIS) und Turkmenistans zu den SCO-Treffen eingeladen.

18 Kissinger 2011.

19 Ein Indiz für Chinas Ambitionen ist auch der kürzlich mit Nicaragua abgeschlossene Vertrag über die Errichtung eines weiteren Kanals zwischen Atlantik und Pazifik, dem sogenannten Nicaragua-Kanal, durch eine chinesische Firma. Das Investitionsvolumen wird auf 40 Mrd. US-Dollar geschätzt. Seit 2009 hat China die USA auch als wichtigster Handelspartner Brasiliens abgelöst. Tendenziell gerät die Monroe-Doktrin - Amerika den Amerikaner - durch den wachsenden Einfluss Chinas in Lateinamerika in Gefahr.

20 Inzwischen wickelt Australien rund 30 Prozent seines Außenhandels mit China ab. Zudem wurde zwischen China und Australien im April 2013 eine direkte Konvertibilität zwischen dem chinesischen Renminbi und dem australischen Dollar vereinbart, das heißt der US-Dollar hat als Rechnungseinheit im chinesisch-australischen Außenhandel ausgedient; Murdoch, Chambers 2013. 
zur Weltmacht vorprogrammiert. ${ }^{21}$ Keine der traditionellen etablierten Mächte wird seine Positionen freiwillig zugunsten Chinas räumen wollen. So unterhält der US-Kongress einen Ausschuss, die U.S. - China Economic and Security Review Commission, der sich laufend ausschließlich mit Fragen der Entwicklung Chinas und deren Konsequenzen für die Sicherheit und die US-Wirtschaft beschäftigt. Das "asiatische Jahrhundert ", das von zahlreichen Autoren ${ }^{22}$ für das 21. Jahrhundert prognostiziert wird, könnte eines neuer wirtschaftlicher und politischer Spannungen werden. Grundlage all dieser Prognosen und Zukunftsszenarien ist jedoch, dass China seine wirtschaftliche Entwicklung ungebremst fortsetzt. Entsprechend einer aktuellen Studie der Weltbank ${ }^{23}$ muss China sich jedoch einem gewaltigen Strukturwandel seiner Wirtschaft und Gesellschaft stellen, damit es nicht in die sogenannte Mittelschichtenfalle (Middle-Income Trap) ${ }^{24}$ eines sich entwickelnden Landes fällt. Bisher hat China vorwiegend ein ressourcenintensives Wachstum betrieben, wobei die weltweit exorbitant niedrigen Lohnkosten ${ }^{25}$ einen wesentlichen Wettbewerbsvorteil in Bezug auf die Standortwahl insbesondere auch ausländischer multinationaler Konzerne darstellte.

\subsection{Chinas Öffnung im Zuge der Globalisierung}

Im Zuge des in den 1990er Jahren einsetzenden Schubes der Globalisierung wurden Produktionsstandorte vieler multinationaler Unternehmen aus Ländern der Triade in großem Umfang nach China verlagert. Was in Europa nach dem Zusammenbruch der Sowjetunion als Verlagerung von Produktionen aus Westeuropa nach Osteuropa stattfand, vollzog sich in Asien mit der Öffnung Chinas vorrangig zunächst zwischen den USA und anderen asiatischen Anrainerstaaten, insbesondere Japan. Durch die Politik der offenen Tür, die Ende der 1970er Jahre von Deng Xiaoping eingeleitet wurde, konnten ausländische Unternehmen in den speziell dafür eingerichteten Sonderwirtschaftszonen ${ }^{26}$ Produktionsstätten errichten und von dort aus

21 China hatte bereits nach dem Ende des Zweiten Weltkriegs militärische Auseinandersetzungen mit den USA - im Koreakrieg, in Indien, Vietnam - und mit der damaligen Sowjetunion. Derzeit gibt es wegen der Senkaku-Inseln beziehungsweise Diaoyu(tai)-Inseln mit Japan erhebliche politisch-militärische Differenzen. Die bisher unter japanischer Kontrolle befindlichen Inseln haben wegen vermuteter Ölvorkommen sowie bezüglich der Nutzung der Fischereirechte innerhalb der 200-Meilen-Wirtschaftszone auch eine wirtschaftliche Bedeutung erlangt.

22 Pilny 2005.

23 Weltbank 2013.

24 Eichengreen et al. 2013.

25 Freeman 1995.

26 »Die ersten fünf Sonderwirtschaftszonen in China wurden in den Provinzen Guangdong, Fujian und Hainan eingerichtet. Während in Fujian nur die Stadt Xiamen zur Sonderwirtschaftszone wurde, war es auf Hainan die gesamte Inselprovinz, die zur Sonderwirtschaftszone erklärt wurde. In Guangdong wurden Sonderwirtschaftszonen in den Städten Shenzhen, Zhuhai und Shantou eingerichtet. «Siehe hierzu www.china9.de/wirtschaft/so nderwirtschaftszonen.php (Zugriff vom 18.12.2013).

Leviathan, 42. Jg., 1/2014 
die produzierten Waren in ihre weltweiten Absatzmärkte exportieren, insbesondere auch in ihre Heimatländer Japan, Taiwan, die USA und die Staaten Westeuropas. Später wurden dann die Sonderwirtschaftszonen entgrenzt und Restriktionen aufgehoben, sodass sich jetzt ausländische Unternehmen in ganz China ansiedeln können. China orientiert sich seit dieser Öffnung am Modell des exportorientierten Wirtschaftswachstums, ${ }^{27}$ das zuvor Länder wie Japan, Südkorea, Taiwan, Singapur und Hong Kong bereits erfolgreich umgesetzt hatten. Allerdings nutzte China ausländische Investoren in neuartiger Weise direkt für die Modernisierung der eigenen Wirtschaft, um sich den Zugang zu deren praktischem Wissen mittels der Firmenverbindungen (Joint Ventures) zu verschaffen und durch die Beschäftigung chinesischer Arbeitskräfte auf allen Ebenen der Produktionsstätten auch entsprechende Kompetenzen anzueignen. Parallel dazu wurden chinesische Studenten in die USA und nach Westeuropa geschickt, die dort an den Spitzenuniversitäten ihre Ausbildung absolvierten. Dadurch konnte China sich einen Humankapitalstock bilden, der mit denen der führenden Länder konkurrenzfähig war. Ein Teil davon machte danach in den Studienländern Karriere, ein anderer kehrte nach China zurück und unterstützte dort den Aufbau einer leistungsfähigen Wirtschaft auf Basis der erlernten westlichen Standards.

Diese asymmetrische Kooperation, bei der die ausländischen Unternehmen aufgrund der für sie günstigen Löhne sowie weiterer Standortvorteile wie Steuerbefreiungen, niedriger Umweltstandards und Marktzutritt zum chinesischen Binnenmarkt hohe Gewinne erzielen konnten, führte zugleich dazu, dass sie zu Lehrmeistern ihrer chinesischen Partner wurden. Da die chinesischen Unternehmen, wenn sie genug gelernt hatten, in der Regel solche Zusammenarbeiten beendeten, erwies sich eine nachhaltige Kooperation später als schwierig. Die hohen Gewinne dienten somit als Köder für einen ungewollten Wissenstransfer. In zahlreichen Fällen blieben diese Kooperationen daher nur Bündnisse auf Zeit.

Die exportorientierte Entwicklungsstrategie führte zu einem schwelenden Handelskonflikt mit den USA, die zuerst das Hauptziel der Exportoffensive aus China waren. Ungefähr grob die Hälfte der chinesischen Exporte ins Ausland stammen von chinesischen Niederlassungen ausländischer Konzerne, die im Zuge der Verlegung von Betriebsteilen Fertigungsstätten in China errichteten oder Teile der Fertigung an chinesische Subunternehmen auslagerten. Zu einem erheblichen Teil finden diese Produktionen, wie gesagt, in Form von Joint Ventures mit chinesischen Unternehmen statt. Die Errichtung gemeinsamer Unternehmensgründungen, in der Regel mit chinesischen Staatsbetrieben, ${ }^{28}$ soll aus Sicht der chinesischen Regierung einen nachhaltigen Technologie- und Wissenstransfer sicherstellen, sodass die chinesische

\section{Balassa 1981; Melo, Robinson 1990; Giles, Williams 1999.}

28 So entfielen von 42 chinesischen Unternehmen bei den Fortune Global 500 im Jahr 2010 39 auf Staatsunternehmen, und nur drei hatten private Eigentümer. Inzwischen - im Jahr 2012 - ist die Anzahl der chinesischen Unternehmen in den Fortune Global 500 bereits auf 73 angestiegen, das heißt es gibt eine immer größere Zahl von chinesischen Staatsbetrieben, die, gemessen an ihrer Umsatzgröße, international ganz weit vorne stehen. Hinter den USA mit 132 hat China noch vor Japan mit 68 bereits den zweiten Rang im 
Wirtschaft sich Schritt für Schritt zu einer eigenständigen leistungsfähigen Unternehmensstruktur fortentwickeln kann und die Modernisierung der chinesischen Wirtschaft möglichst rasch vonstatten geht. Mithin wurden ausländische multinationale Konzerne zu den wichtigsten Treibern der chinesischen Wirtschaftsentwicklung. Das Entwicklungsmodell Chinas ist also ganz eigen, anders als dasjenige Japans und Südkoreas, deren Industriepolitik auf der massiven Förderung von national champions durch den Staat basierte (in Japan den Keiretsus ${ }^{29}$ beziehungsweise in Südkorea den Jaebeols ${ }^{30}$ ).

Beispiel wäre etwa die SAIC (Shanghai Automotive Industry Corporation), die ein Joint Venture mit Volkswagen und später auch General Motors eingegangen ist. Für die ausländischen Unternehmen bietet das den Vorteil, auch den chinesischen Markt mit ihren Fahrzeugen zu erschließen. Lange Jahre war der VW Santana, der als erstes westliches Automodell in China gefertigt wurde, die Standardlimousine, bevor man den Markt für andere Hersteller aus den USA, Japan, Südkorea und Europa öffnete. Damit konnte die chinesische Staatsführung erreichen, dass nicht nur ein veraltetes Modell wie der Santana, sondern die aktuellsten Modelle der ausländischen Hersteller nun in China gefertigt und auf einem wachsenden Markt verkauft wurden. Inzwischen bauen VW und BMW dort eine Reihe von Werken aus und haben mit der Herstellung von Motoren begonnen, und im November 2013 schloss auch Daimler Benz einen Vertrag über Produktion und Motorenbau in Peking mit der chinesischen Beijing Automotive Industry Holding (BAIC). ${ }^{31}$ Martin Winterkorn, der Vorstandsvorsitzende von VW, wurde zu einem Top-Berater der chinesischen Staatsführung ernannt. ${ }^{32}$ Es stellt sich die Frage, ob der alte Spruch aus den USA, "what is good for General Motors, is good for America", in abgewandelter Form auch für VW und Deutschland Gültigkeit hat. Die chinesische Staats-

Jahr 2012 eingenommen. Deutschland liegt mit 32 gemeinsam mit Frankreich auf Rang 4. Vgl. The Economist 2011; Fortune Global 500 (siehe Appendix). Chinas Staat kontrolliert mithin insbesondere die chinesischen Großunternehmen in Form von Staatsunternehmen. Private Unternehmen wie Geely oder Hutchinson Whampoa sind dagegen nur von marginaler Bedeutung. Zudem sind sie ebenfalls auf das Wohlwollen der chinesischen Partei- und Staatsführung angewiesen, wenn sie in China erfolgreich ihre Geschäfte machen wollen.

29 Keiretsu (wörtlich: Reihe, Linie) bezeichnet japanische Zusammenschlüsse von Unternehmen, auch »wirtschaftliche Verbundgruppen « genannt.

30 Jaebeol (dt. reiche Sippe, von jae Reichtum oder Besitz und beol Klan oder Sippe) ist der koreanische Begriff für ein großes Familienunternehmen, das meist aus verschiedenen Sparten besteht, also ein Mischkonzern (Konglomerat) ist.

31 Siehe Automobilproduktion 2013. »Gemeinsam mit den Partnern baut Volkswagen sieben neue Werke und investiert in den nächsten Jahren insgesamt mehr als zehn Milliarden Euro«; Handelsblatt 2013 a. Vgl. auch Handelsblatt 2013 b; Handelsblatt 2013 c.

32 »Zum sogenannten Global CEO Advisory Council gehören dem Bericht zufolge neben Winterkorn internationale Firmenchefs wie General-Electric-Chef Jeffrey Immelt und Disney-Boss Robert Iger aus den USA oder Carrefour-Chef Georges Plassat aus Frankreich. Winterkorn ist der einzige deutsche Vertreter und Automanager der Beraterrunde. Der VW-Chef ist der bestbezahlte Vorstandschef Deutschlands «; Der Spiegel 2013.

Leviathan, 42. Jg., 1/2014 
führung plant jedenfalls, so heißt es, umgekehrt in den kommenden Jahren den Weltmarkt für PKWs von China aus zu erobern.

Beispiele aus dem Anlagenbau zeigen dasselbe Muster. Die großen Turbinen zur Stromerzeugung im Rahmen des Drei-Schluchten-Staudamm-Projekts wurden von Firmen wie ABB, Siemens und General Electric in China in Kooperation mit einer chinesischen Partnerfirma gefertigt. Seitdem ist China in der Lage, solche Turbinen selber herzustellen und zu vertreiben. Entsprechend wurde der Wettbewerb ausländischer Firmen um Aufträge bei Hochgeschwindigkeitszügen eingesetzt, um den Technologietransfer auch in diesem Bereich zu ermöglichen. Der massive Ausbau der Hochgeschwindigkeitsnetze in China nach 2008 erfolgte dann durch chinesische Unternehmen. Diese bieten inzwischen ihre Züge nicht nur in Thailand und Birma, sondern auch in Kalifornien an. ${ }^{33}$ In diesem Jahr wurde darüber hinaus beim Besuch des chinesischen Ministerpräsidenten Li Keqiang in Osteuropa eine Reihe von Vereinbarungen zum Bau von Hochgeschwindigkeitsstrecken durch chinesische Firmen in Ungarn und Serbien getroffen. Ebenso hat Großbritannien einer Kooperation beim Bau einer Strecke in Großbritannien zugestimmt. ${ }^{34}$

Ein ähnliches Vorgehen zeigt sich beim Ausbau der chinesischen Mobilfunknetze: Es gibt heute leistungsfähige chinesische multinationale Konzerne wie Lenovo, HTC, Huawei, die den anderen vorwiegend asiatischen und amerikanischen Konzernen (neben Nokia und Sony-Ericsson) Konkurrenz auch auf deren Heimatmärkten machen. Siemens hat dieses Geschäftsfeld bereits vollständig aufgegeben. Nokia, einst Weltmarktführer bei Mobiltelefonen, hat sich vor kurzem ebenfalls aus diesem Bereich zurückgezogen und seine Tochterfirmen an Microsoft verkauft. ${ }^{35}$ Auch im Bereich der Elektronik und Halbleiterfertigung sowie im Bereich der Telekommunikation hat China führende Unternehmen aus dem Ausland angelockt. Foxconn $^{36}$ ist zum weltweit führenden OEM-Fertiger ${ }^{37}$ für fast alle großen internationalen Elektronikkonzerne wie Sony, Samsung, LG, HP, Apple etc. aufgestiegen.

33 »Das chinesische Hochgeschwindigkeitsnetz wird Ende 2012 über 10.000 Kilometer lang sein und Reisenden eine schnelle, sichere und preisgünstige Alternative zum Flugzeug bieten «; Xinhua 2012. Vgl. Handelsblatt 2013 d.

34 Rheinpfalz 2013.

35 Die Welt 2013 b.

36 »Foxconn Electronics Inc. ist eine Tochtergesellschaft des taiwanischen Unternehmens Hon Hai Precision Industry Co., Ltd. und der Name, unter dem das Unternehmen auf dem Markt auftritt. Das Unternehmen wurde 1974 von Terry Gou als Hersteller von Kunststoffprodukten gegründet. Seit 1991 ist das Unternehmen an der taiwanischen Börse notiert. Heute ist es einer der größten Fertigungsbetriebe für elektronische Produkte weltweit «; http://de.wikipedia.org/wiki/Foxconn (Zugriff vom 19.12.2013). Im Mai 2013 beschäftigte Foxconn rund 1,2 Millionen Personen.

37 »Unter einem Erstausrüster bzw. Original-Equipment-Manufacturer (abgekürzt OEM, engl. für Originalausrüstungshersteller) versteht man einen Hersteller von Komponenten oder Produkten, der diese in seinen eigenen Fabriken produziert, sie aber nicht selbst in den Einzelhandel bringt «; http://de.wikipedia.org/wiki/Erstausr\%C3\%BCster (Zugriff vom 19.12.2013). 
Diese haben China inzwischen zum größten Exporteur von Waren der Informations- und Kommunikationstechnologie werden lassen. ${ }^{38}$

\section{Marktwirtschaft mit chinesischen Merkmalen}

China hat sich allerdings niemals dem Modell einer dezentralen Marktwirtschaft westlicher Prägung verschrieben. Der Staat und die durch ihn vertretene Parteibürokratie waren immer bestrebt, den staatlichen Einfluss sowohl auf die chinesischen Staatsbetriebe als auch auf ihre westlichen Kooperationspartner aufrechtzuerhalten. ${ }^{39}$ Dies wird mit dem dafür verwendeten Begriff einer sozialistischen Marktwirtschaft mit chinesischen Merkmalen umschrieben. Letztendlich sollte die KP Chinas immer das letzte Wort bei allen wichtigen Aktivitäten auch ausländischer Unternehmen in China haben. Nach einer Studie der Chinese Enterprise Confederation, des chinesischen Unternehmerverbands, waren von den 500 größten chinesischen Unternehmen der ausgewiesenen 75 Wirtschaftszweige in 29 Wirtschaftszweigen ausschließlich Staatsunternehmen tätig. In zehn weiteren Wirtschaftszweigen existierten nur ganz wenige Privatunternehmen, die dort kaum eine wichtige Rolle einnahmen. Insgesamt kontrollierte der Staat rund 85 Prozent des gesamten Anlagevermögens in den über Staatsunternehmen weitestgehend kontrollierten Wirtschaftszweigen..$^{40}$ Der Staat befindet sich daher trotz aller Reformen weiterhin generell in der Rolle des Hegemons gegenüber allen Vertretern der Wirtschaft, ob sie nun staatliche oder private chinesische Unternehmen sind oder eben auch ausländische. Alle haben sich letztendlich den Weisungen der chinesischen Staatsführung zu beugen.

Obwohl sich der Staat sukzessive aus zahlreichen Bereichen der chinesischen Wirtschaft zurückgezogen hat, sodass heute in etwa die Hälfte der Wertschöpfung Chinas von Privatunternehmen erbracht wird, kontrolliert er weiterhin entsprechend die andere Hälfte der Wirtschaftsleistung direkt oder indirekt über die in seinem Besitz befindlichen Staatsunternehmen. ${ }^{41}$ Diese folgen einer strategischen Planung der Zentralregierung, die mittels dieser Staatsbetriebe ihre industriepolitischen Ziele umsetzen kann. Daneben kann die chinesische Führung über die gewaltigen Mittel aus ihren Leistungsbilanzüberschüssen mithilfe ihres Staatsfonds $\mathrm{CIC}^{42}$ auch eine Politik der Direktinvestitionen im Ausland betreiben, um sich den notwendigen Einfluss auf ausländische Unternehmen und deren technologisches

38 Saxenian 2006.

39 Walter, Howie 2012.

40 Siehe hierzu The Economist 2011.

41 Huang 2008.

42 »CIC - China Investment Corporation was established in 2007 with approximately US $\$ 200$ billion of assets under management. As of August 2013, the CIC has 575.2 billion in assets under management «; siehe http://en.wikipedia.org/wiki/China_Investment_Cor poration (Zugriff vom 19.12.2013). 
Know-how zu sichern. ${ }^{43}$ So ist Deutschland inzwischen zu einem wichtigen Ziel für chinesische Direktinvestitionen geworden. ${ }^{44}$

Das hat jedoch auch die Korruption innerhalb des chinesischen Partei- und Staatsapparats gefördert. Um bestimmte Genehmigungen zu erlangen, ist ein komplexes System der Korruption auf allen Ebenen der chinesischen Partei- und Staatsführung entstanden. Nur wer über ausreichende Beziehungen, »Guanxi « 45 , zu den richtigen Vertretern verfügt, wird letztendlich in China wirtschaftlich erfolgreich sein können. Der Prozess gegen den ehemaligen Minister für das Eisenbahnwesen, Liu Zhijun, wegen massiver Bestechlichkeit im Amt, der mit einem Todesurteil auf Bewährung endete, ${ }^{46}$ zeigt deutlich, wie weit Korruption bis in die Führungsspitze von Partei und Staat vorgedrungen ist. Ein anderer prominenter Fall ist der des Milliardärs Lai Changxing, der sich durch Korruption und Schmuggel von Luxuswaren aus dem Ausland zum Dollar-Milliardär emporarbeiten konnte. ${ }^{47}$ Mit dem Erfolg der chinesischen Wirtschaft nahm auch die Korruption in Partei und Staat immer größere Ausmaße an.

Dies hat zuletzt zu einer Reihe von politischen Kampagnen der chinesischen Partei- und Staatsführung gegen Bestechung geführt, da offenbar das, was zunächst nur als »Schmiermittel « für die rasche Genehmigung und Durchführung von wirtschaftlichen Projekten durchaus für alle beteiligten Akteure nützlich war, ${ }^{48}$ sich nun zunehmend als wirtschaftlich bedeutsamer Faktor erweist, der die Entwicklung bremst. ${ }^{49}$ Wie ernst die Lage derzeit selbst von Seiten der offiziellen chinesischen Staatsführung eingeschätzt wird, zeigt die Rede zur Lage der Nation des scheidenden chinesischen Staatspräsidenten Hu Jintao auf dem Parteikongress im November 2012, bei dem die Macht an die neue Partei- und Staatsführung offiziell übergeben worden ist. ${ }^{50}$ Waren viele westliche Manager zuvor noch voll des Lobes, dass man sich in China nicht wie in westlichen Demokratien mit einer Fülle heterogener Gruppeninteressen einschließlich der von NGOs durch ein komplexes Rechtssystem hindurcharbeiten musste, sondern durch Gefälligkeiten schnell zu seinen Genehmi-

\section{Giesen 2013.}

44 Munich Innovation Group 2013.

45 »Guanxi bezeichnet das Netzwerk persönlicher Beziehungen, von dessen Wirken in China kaum eine Entscheidung unbeeinflusst bleibt. Verträge und Absprachen werden in vielen Fällen nur als eine Richtschnur gesehen, von der im Zweifelsfall abgewichen werden darf. Guanxi ist ein vielschichtiges Wort. Im Wörterbuch ist es mit Verbindung, Beziehung übersetzt «; siehe http://de.wikipedia.org/wiki/Guanxi (Zugriff vom 19.12.2013).

46 Süddeutsche Zeitung 2013.

47 Er hatte unter anderem einen großen Teil der Provinzregierung von Fukian sowie den Vizebürgermeister und Chef der Zollbehörde der Hafenstadt Xiamen wie auch den stellvertretenden Minister für öffentliche Ordnung in Beijing auf seiner Bestechungsliste stehen; siehe Eckholm 2000.

48 Bellow 2003.

49 Fisman, Migual 2008.

50 Reuters 2012. 
gungen kam, so wird die Korruption nun immer teurer und damit unbezahlbar oder zumindest zu einem wesentlichen Ärgernis. Jetzt bekommen auch die ausländischen multinationalen Unternehmen Druck von der Parteispitze, Korruption innerhalb Chinas zu unterlassen. ${ }^{51}$

Aufgrund der fortdauernden Rechtsunsicherheit konnte man nie sicher sein, ob nach Zahlung eines Bestechungsgelds nicht zu einem späteren Zeitpunkt weitere Forderungen auftauchen. All dies wird nun zunehmend als erhebliche Einschränkung empfunden und schwächt die Standortqualität Chinas insbesondere für ausländische Hersteller, die mit dieser Mentalität nicht umgehen können. Die ausländischen Hersteller haben freilich die Option, das Land wieder zu verlassen, eine Option, von der inzwischen kleine und mittelständische Unternehmen, die ihr Glück in China gesucht hatten, zunehmend Gebrauch machen. Fehlende Rechtssicherheit und Schutz vor staatlicher Willkür, fehlender Patent- und Markenschutz und Behinderungen beim Marktzugang haben den Optimismus der Investoren getrübt. So hatten sich viele enttäuschte ausländische Unternehmer das gelobte Land China der mehr als einer Milliarde Verbraucher und ungesättigter Märkte nun doch nicht vorgestellt: Nach anfänglicher Euphorie hat nun eine Phase der Ernüchterung begonnen.

In den Ländern der westlichen Welt wächst aufgrund der seit Ausbruch der weltweiten Wirtschafts- und Finanzkrise außerordentlich hohen Arbeitslosigkeit der Druck der Öffentlichkeit, den massiven Arbeitsplatzverlust durch Produktionsverlagerungen in ausländische Standorte nicht ungebremst fortzusetzen. Zugleich sind nun auch die Arbeitskosten in den USA und Europa relativ zu denen in China gesunken, da sie in den chinesischen Küstenprovinzen rasant gestiegen sind. Sich weiter westlich in den chinesischen Binnenprovinzen anzusiedeln, stößt auf Probleme unzureichender Verkehrsinfrastrukturen und Energieversorgung und des Mangels an qualifizierten Arbeitskräften. Der demographische Wandel aufgrund der Ein-Kind-Politik macht sich in China bemerkbar, weil es nun weniger junge Arbeitskräfte gibt und auch weniger Menschen als noch vor einigen Jahren vom Land in die Städte abwandern. ${ }^{52}$

\subsection{Rückschlag für Chinas Expansionsstrategie}

In den USA und Großbritannien, die Vorreiter dieser Entwicklung waren, ist man sich schmerzhaft bewusst geworden, dass sich progressive Leistungsbilanzdefizite ohne zunehmende Warenexporte aus ihren Ländern auch mittelfristig nicht abbauen lassen. Der Weg in eine Dienstleistungsgesellschaft, die sich weitestgehend ohne einen industriellen Kern erfolgreich im globalen Wettbewerb behaupten könnte, erweist sich jetzt als eine Bedrohung für die wirtschaftliche Stabilität ihrer Länder. Barack Obama hat deshalb seiner Regierung das Ziel gesetzt, das Außen-

51 »According to Beijing-based private research firm Anbound Group, of some 500,000 corruption cases investigated in China from 2000 to 2009, about 64 percent were linked to international trade and foreign businesses «; Weihua 2010. Siehe auch China Briefing 2013.

52 Hvistendahl 2012.

Leviathan, 42. Jg., 1/2014 
handelsvolumen der USA bis zum Jahr 2016 zu verdoppeln. Parallel dazu soll eine Strategie der Reindustrialisierung ${ }^{53}$ die Voraussetzungen schaffen, dass wieder Industriegüter in größerem Maße als bisher exportiert werden können. Der erwähnte, teilweise rasante Lohnkostenanstieg in China trägt zur Erreichung dieses Ziels bei, denn insbesondere amerikanische Hersteller kehren inzwischen aus China wieder in die USA zurück. ${ }^{54}$

Auch europäische und hier vor allem deutsche Hersteller leiden unter den sich verschlechternden Standortbedingungen in China und anderen Billiglohnstandorten und holen ihre zuvor verlagerten Produktionen zurück. ${ }^{55}$ So lässt sich das interne Wissen sicherer vor einem unerwünschten Abfluss schützen. Vor Verlusten durch Markenpiraterie versucht man sich zugleich durch Techniken wie RFID-Markern $^{56}$ oder kDNA-Kennzeichnung ${ }^{57}$ seiner Produkte insbesondere bei Ersatzteilen zu schützen. Da die Innovationszyklen verkürzt werden, was eine größere Nähe zum Kunden erforderlich macht, erweisen sich lange Transportwege und Lieferzeiten als Hemmnis für die entsprechend notwendige Flexibilität. Dies gilt sogar im Bereich der Textilindustrie. Modeketten wie Zara und H\&M wechseln ihr Produktsortiment bereits siebenmal im Jahr. Dies lässt sich mit Fertigungen in China oder anderen entfernten Produktionsstandorten und den damit verbundenen zahlreichen Unwägbarkeiten nicht realisieren.

China, das sollte hier zunächst gezeigt werden, hat im Zuge seiner rasanten wirtschaftlichen Entwicklung der letzten Jahre nicht nur Fortschritte erzielen können, wie dies die makroökonomischen Daten suggerieren könnten, sondern es haben sich zahlreiche strukturelle Defizite aufgebaut, die die weitere Entwicklung hemmen werden. China wird nicht umhinkommen, wesentliche strukturelle Reformen umzusetzen, die über die Bereiche Umwelt und Infrastruktur hinausgehen: Es geht durchaus um tiefgreifendere gesellschaftliche beziehungsweise gesellschaftspolitische Reformen. Das Verhältnis von Staat und Wirtschaft und von Wirtschaft und Gesellschaft muss neu kalibriert werden, wenn China sich weiter an das Niveau der westlichen Welt (einschließlich der asiatischen entwickelten Industrieländer) heranarbeiten will. $\mathrm{Ob}$ dies am Ende gelingen wird, muss sich noch erweisen.

53 Euler-Hermes 2013.

54 Boston Consulting Group 2011; Evolve 2012.

55 Kinkel 2012.

56 »RFID (engl. radio-frequency, >Identifizierung mit Hilfe elektromagnetischer Wellen $<$ ) ermöglicht die automatische Identifizierung und Lokalisierung von Gegenständen und Lebewesen und erleichtert damit erheblich die Erfassung von Daten (umgangssprachlich auch Funketiketten genannt)«; http://de.wikipedia.org/wiki/RFID (Zugriff vom 19.12.2013).

57 kDNA: künstliche DANN; siehe http://de.wikipedia.org/wiki/K\%C3\%BCnstliche_DNA (Zugriff vom 19.12.2013). 


\section{Warum Keynes versus Hayek?}

Seit Langem gibt es in den Wirtschaftswissenschaften eine Grundsatzdebatte, die zwischen keynesianischen und neoliberalen Ökonomen ausgetragen wird. ${ }^{58}$ Dabei geht es um grundsätzliche Fragen, wie das Gesellschaftsmodell und das Wirtschaftssystem organisiert werden sollten.

Keynes steht dabei für eine starke Stellung des Staates, der mittels eines Staatsinterventionismus das unvermeidliche Marktversagen unvollkommener Märkte kontrollieren, regulieren und kompensieren muss. Nur durch das Schaffen eines Ordnungsrahmens sowie gezielte Intervention bei akutem Marktversagen kann der Kapitalismus am Leben erhalten werden. Ansonsten droht der Rückfall in totalitäre Regime in dem Moment, in dem der Kapitalismus seine Legitimation gegenüber der Mehrheit der Gesellschaft einbüßt. Keynes träumte also weniger von einem utopischen Reich unbeschränkter Freiheit, stattdessen ging es ihm darum, die Auswüchse eines Systems, das den Keim einer Selbstzerstörung in sich trägt, so weit zu bändigen, dass das System überlebensfähig ist und nicht kommunistischen oder nationalsozialistischen Experimenten anheimfällt. Er verstand sich jedoch selbst als Liberalen ${ }^{59}$ in der Tradition von John Stuart Mill ${ }^{60}$.

Im Gegensatz hierzu steht eine sich auf Hayek berufende Richtung, die Staatsinterventionismus als schädlichen Dirigismus brandmarkt, der gerade die ansonsten potenziell mögliche Effizienz eines freien Marktgeschehens in ihrer Vitalität hemmt und damit den Wohlstand der Gesellschaft schmälert. Für den späten Hayek war staatliche Intervention immer auch Anmaßung von Wissen, ${ }^{61}$ die letztendlich aufgrund der »Arroganz der Mächtigen « Schaden stiftet. Er dachte dabei nicht nur an den wirtschaftlichen Schaden, der sich in Euro oder Dollar messen lässt, sondern eben auch an den Verlust an dem, was er unter individueller Freiheit zu einer selbstbestimmten Lebensführung verstand, denn für ihn gab es keine "Gesellschaft ", wie seine Schülerin Margret Thatcher es später ausdrücken sollte, sondern nur Ansammlungen von selbst zusammengestellten Verbindungen. Wirtschaftliche Freiheit und persönliche Freiheit sind bei Hayek eng miteinander verbunden, weshalb er den Sozialismus jeder Couleur als Hauptfeind der Freiheit ausmachte. Zugleich fürchteten die Hayekianer die schleichende Erosion des Liberalismus auch in den westlichen Demokratien, die eben durch einen »ausufernden "Sozialstaat und staatliche Regulierung bis hin zur Wirtschaftsplanung die Grundlagen einer freien Marktwirtschaft und eines ungebändigten Unternehmertums zu unterminieren drohte. ${ }^{62}$ Makroökonomische Steuerung der Wirtschaft, die sich auf Keynes berief, war damit aus Sicht neoliberaler Vertreter immer schon verdächtig und sollte durch Deregulierung der Märkte und Abbau staatlicher Einflüsse wie Privatisierung staatlicher

58 Wapshot 2011.

59 Keynes 1991 [1925].

60 Mill 2003 [1859].

61 Hayek 1973.

62 Hayek 1943.

Leviathan, 42. Jg., 1/2014 
Unternehmen bekämpft werden. Besonders Milton Friedman und die Politik unter Margret Thatcher und Ronald Reagan verschrieben sich diesen Zielen, die Hayek als Repräsentanten für ihr eigenes politisches Denken reklamierten.

Diese Kontroverse hat auch durchaus für China seine Relevanz, das eine andere Entwicklungsgeschichte als der Westen durchlaufen hat. China hat sich nach dem Sieg Maos und der KP Chinas über die Kuomintang unter Chiang Kai-shek zunächst am Wirtschaftsmodell der UdSSR unter Stalin orientiert. Als die damalige Sowjetunion sich dann aufgrund zunehmender Probleme mit der sozialistischen Planwirtschaft unter Chruschtschow zu marktwirtschaftlichen Reformen entschloss, kam es zum Bruch zwischen der Sowjetunion und der Volksrepublik China. Mao warf Chruschtschow Revisionismus vor. Es ging dabei letztendlich aber um den weltweiten Führungsanspruch im kommunistischen Lager, dem auch wirtschaftliche Interessengegensätze zugrunde lagen. China benötigte damals in großem Umfang wissenschaftliches und technisches Know-how der Sowjetunion, um den Aufbau des Landes vorantreiben zu können. Chruschtschow reagierte jedoch am Ende damit, dass er sämtliche russischen Experten, die China beim wirtschaftlichen Ausbau unterstützten, aus China abzog. Mao versuchte danach mittels des Großen Sprungs den chinesischen Alleingang, der - wie wir heute wissen - in einem wirtschaftlichen Fiasko endete. ${ }^{63}$ China musste in der Folge erst mühsam wieder wirtschaftlich stabilisiert werden, und die wirtschaftlichen Ambitionen Maos mussten auf ein vernünftiges Maß zurückgeführt werden. Nachdem die alten Kader der KP Chinas dies nach einiger Zeit erreicht hatten und eher dazu tendierten, nun ebenfalls marktwirtschaftliche Reformen in China einzuführen, startete Mao erneut eine Kampagne der großen proletarischen Kulturrevolution mit dem Ziel, den nun wieder erneuerten, am stalinistischen Planwirtschaftsmodell orientierten Weg der chinesischen Wirtschaft hin zu einer kommunistischen Wirtschaft in Gang zu setzen. Auch dieser Versuch endete in einem wirtschaftlichen und menschlichen Fiasko.

Erst nach dem Tod Maos und der Ausschaltung der »Viererbande « konnten sich dann unter der Führung von Deng Xiaoping Reformer durchsetzen, die China weg von der Planwirtschaft stalinistischer Prägung hin zu einer Marktwirtschaft mit chinesischen Merkmalen führten. Das bedeutete insbesondere auch die Öffnung Chinas zum Westen. Die chinesische Führung in Partei und Wirtschaft hatte begriffen, dass sie vom Westen mehr lernen konnte als von ihren ehemaligen Verbündeten in der Sowjetunion. Wollte China nicht weiter hinter die wirtschaftliche Entwicklung der übrigen Welt zurückfallen, dann musste es sich radikal auf den Import westlicher Technologie und westlichen Know-hows einlassen. Das Vehikel dazu war

63 »Bedingt durch die Zwangskollektivierung der Landwirtschaft, die Zusatzbelastung der Bauern durch Arbeiten an Infrastruktur- und Industrialisierungsprojekten und eine Binnenmigration der Landbevölkerung in die Städte sanken die landwirtschaftlichen Erträge von 1959 bis 1961. Gleichzeitig wurden die vom Staat als Steuer und für den Export erwarteten Getreideabgaben stark herauf- und mit Zwangsmaßnahmen durchgesetzt. Die Zahl der Opfer dieser Hungersnot wird auf 15 bis 45 Millionen Menschen geschätzt. Damit handelt es sich um die größte Hungerkatastrophe in der Geschichte der Menschheit «; http://de.wikipedia.org/wiki/Gro\%C3\%9Fer_Sprung_nach_vorn (Zugriff vom 19.12.2013). 
die Errichtung der Sonderwirtschaftszonen, die aus chinesischer Sicht als Labor für wirtschaftspolitische Experimente dienten.

Der besondere Clou bestand eben für China darin, dass es den ausländischen Unternehmen der kapitalistischen Welt den kontrollierten Zugang zu China und seinem gewaltigen Potenzial an billigen Arbeitskräften und Ressourcen bot, zugleich aber durch die Kontrolle von Partei und Staat diesen Unternehmen Zügel anlegte und sie in die gewünschte Richtung für die Entwicklung einer modernen chinesischen Wirtschaft lenkte. Die hohen Gewinne, die ausländische Unternehmen dabei erzielen konnten, waren das Lockmittel. Der damit gleichzeitig verbundene freiwillige und unfreiwillige Wissenstransfer westlicher multinationaler Unternehmen lieferte für China die gewünschte Entwicklungserleichterung als nachhaltige Dividende. Man muss schon mit der chinesischen Denkweise des Ying und Yang vertraut sein, um diesen scheinbaren ideologischen Salto Mortale der post-maoistischen Ära würdigen zu können. Dieses Zweckbündnis mit dem Klassenfeind war jedenfalls ein überraschender Schritt, der China vor einem ähnlichen Schicksal wie das der Sowjetunion bewahrt hat.

Durch die Politik der Annäherung an die USA und den Abbau außenpolitischer Konflikte insbesondere in Südost- und Ostasien (etwa durch den Frieden in Vietnam) wurde es China ermöglicht, sich mit massiver Unterstützung des Westens zu reformieren. Hierbei spielte eine technokratische Führungselite die entscheidende Rolle, Pragmatismus ersetzte das radikale menschenverachtende Ideologieprogramm. Damit knüpfte man zugleich an alte konfuzianische Traditionen der chinesischen Geschichte an. Es ist eben diese Synthese aus chinesischer Tradition und dem Willen zur Absorption des westlichen Modells des Wirtschaftens, die China auf einen Weg der Modernisierung gebracht hat, wobei die Regulierung der Ökonomie durch die Politik erhalten blieb.

Allerdings führte die Beibehaltung der zentralistischen Kontrolle von Wirtschaft und Staat unter der Führung der KP Chinas eben auch zu den unerwünschten Begleitumständen, dass sich zunehmend Spannungen zwischen neuen jungen Eliten und der etablierten Partei- und Staatsführung aufbauten, die ihren symbolisch stärksten Ausdruck in den Demonstrationen für mehr politische und gesellschaftliche Freiheit im Sinne Friedrich von Hayeks auf dem Tienanmen-Platz in Beijing fanden. ${ }^{64}$

Die brutale Unterdrückung dieser Reformbewegung, die mehr Mitbestimmung und auch Selbstbestimmung im Privatleben forderte, durch das chinesische Militär zeigt bis in die Gegenwart das gespaltene Verhältnis der Chinesen zum westlichen Lebensmodell einer freiheitlichen Demokratie mit Gewaltenteilung. Eine der amerikanischen Freiheitsstatue nachgebildete Skulptur auf dem Tienanmen-Platz stand damals als Symbol für diese Forderungen der protestierenden Studenten und jungen Arbeiter. Bis heute bleibt diese Jugendrevolte ebenso wie die Kulturrevolution Maos ein Tabuthema innerhalb Chinas, das nicht öffentlich diskutiert werden kann.

64 Am 3. und 4. Juni 1989 schlug das chinesische Militär im Zentrum Pekings gewaltsam die Proteste der Bevölkerung nieder. 
Aufgrund sozialer und ökologischer Probleme, die die Umwandlung einer Agrarin eine Industriegesellschaft mit sich bringt, wachsen die Spannungen innerhalb Chinas rasant an. Bereits im Jahr 2006 registrierte die Chinesische Akademie für Sozialwissenschaften (CASS) 60.000 Zwischenfälle, bei denen es zu Auseinandersetzungen in China kam. Im Jahr 2007 stieg diese Zahl auf 80.000. Inoffizielle Zahlen für die Jahr 2008 sprechen von 127.000 und für 2010 von 180.000. ${ }^{65}$ Ursache sind lokale Konflikte, die auf ein rücksichtloses Vorgehen staatlicher Stellen zurückgehen sowie auf Korruption und Begünstigung einzelner Privatleute und Unternehmen, die ihre Beziehungen zu lokalen staatlichen Stellen und Funktionären der KP Chinas ausnutzen. Gerade bei der Veräußerung von Landrechten und Enteignungen im Zuge von Großprojekten werden die Interessen und Rechte der lokalen Bevölkerung häufig missachtet: Der Aufstiegsdrang der chinesischen Eliten verträgt sich nicht mit einem westlichen Gewaltenteilungsmodell, in dem die Rechte der Einzelnen gegenüber dem Staat durch unabhängige Gerichte feststellbar sind. Das treibt die soziale Spaltung in China voran und nährt die Unzufriedenheit der Benachteiligten. Es fördert einerseits eine kleine Elitenfraktion, die das westliche Rechtsstaatsmodell einfordert, andererseits aber auch eine Tendenz zum Neo-Maoismus, der aus Nostalgie die gute alte Zeit glorifiziert. ${ }^{66}$ Das führt zu massiven Richtungskämpfen innerhalb der KP Chinas über den zukünftigen Weg der chinesischen Wirtschafts- und Gesellschaftspolitik.

\subsection{Keynesianische Konjunktursteuerung in China}

\section{Die Asienkrise von 1998}

China hatte aufgrund seiner historischen Entwicklung von einer sozialistischen Planwirtschaft hin zu einer mehr marktwirtschaftlichen Orientierung keinerlei Probleme, sich keynesianisches Denken zur Steuerung einer Volkswirtschaft anzueignen. ${ }^{67}$ Die Außenhandelspolitik war bereits ganz im Sinne von Friedrich List am Aufbau und Schutz der eigenen Industrieentwicklung orientiert. Staatliche Wirtschaftslenkung bedeutete dabei nicht nur makroökonomische Steuerung, sondern ebenso die Steuerung einzelner Wirtschaftszweige im Sinne einer keynesianischen

65 Yanqi, Shaohua 2011; Yanqi, Shaohua 2010.

66 Bo Xilai, ein ehemaliges Mitglied des Politbüros der KP Chinas, förderte als Parteichef von Chongqing solche Bestrebungen. »Bo galt als Vertreter und Hoffnungsträger des linken Flügels der Partei. Er strebte eine Rückbesinnung auf die Mao-Zedong-Ära an und bekämpfte eine Ausweitung der marktwirtschaftlichen Orientierung der Parteiführung. Seine Methoden im Kampf gegen Korruption in Chongqing, die von der chinesischen Linken teilweise als nachahmenswertes Modell betrachtet wurden, schlossen auch eine Aushebelung der Gewaltenteilung, direkte Einflussnahme der Exekutive auf die Gerichte und öffentliche >Kampagnen in Anlehnung an die Praktiken der Kulturrevolution ein." Er wurde wegen Beteiligung an einem Mordkomplott seiner Ehefrau sowie Korruption verhaftet und in einem Schauprozess zu lebenslanger Haft von einem chinesischen Gericht verurteilt. Zuvor galt er sogar als aussichtsreicher Kandidat für die Nachfolge von Hu Jintao. Siehe http://de.wikipedia.org/wiki/Bo_Xilai (Zugriff vom 19.12.2013).

67 Allerdings gilt dies erst für die Zeit nach Mao; siehe Cox 2011. 
Investitionslenkung und Planvorgaben im System der sozialistischen Wirtschaftsplanung. Der Markt hatte aus Sicht der chinesischen Staatsführung nur dort eine eigenständige Funktion, wo er sich den vom System gesetzten Rahmenbedingungen unterordnete. Wurden beziehungsweise werden diese unterlaufen, geraten die entsprechenden Akteure in den Bereich der Illegalität und müssen entsprechend mit Sanktionen rechnen. Durch Zahlungen konnten sich Unternehmer jedoch oftmals das Wohlwollen der entsprechenden staatlichen Akteure erkaufen, was letztendlich zu dem rasanten Anstieg der Korruption in China beigetragen hat. Das führte trotz dieser Widersprüche des Systems zu einer relativ stetigen Wachstumsentwicklung Chinas bis zum Ausbruch der weltweiten Wirtschafts- und Finanzkrise 2007.

Als Chinas Wirtschaft während der Asienkrise in der Zeit nach 1998 einen konjunkturellen Einbruch aufgrund sinkender Exporte erfuhr, konnte dem Nachfragerückgang durch die massive Ausweitung der Staatsnachfrage erfolgreich entgegengewirkt werden.

Freilich bestand nicht das Risiko einer massiven Kapitalflucht ausländischen Kapitals aus China, da dieses nicht in Finanzinvestitionen, sondern in Direktinvestitionen festgelegt war. Zudem ließ sich der Wechselkurs des Renminbi gegenüber dem US-Dollar stabil halten, so dass es nicht zu dem vielfach befürchteten Abwertungswettlauf in Asien kam. China wirkte also während der Asienkrise als Stabilisator mithilfe einer keynesianischen Ausgabenpolitik, die jedoch auch auf einer Kreditexpansion und staatlichen Defiziten beruhte. Zugleich konnte das eigene wirtschaftliche Wachstum weitgehend stabil gehalten werden, denn die Asienkrise betraf die chinesischen Hauptabsatzmärkte in den führenden Ländern der Triade kaum, Absatzprobleme gab es lediglich im regionalen Handel insbesondere mit den ASEAN-Staaten. Da China jedoch auch keine frei konvertierbare Währung besaß und der Kapitalverkehr ebenfalls kontrolliert werden konnte, war das Risiko eines externen Schocks in Form massiver Kapitalflucht vergleichsweise gering.

China hat privates Eigentum und privaten Bodenbesitz im Zuge der Bodenreform in den 1950er Jahren zugelassen und weist aufgrund eines nach wie vor weitreichenden Eigentumsmonopols des Staates an Boden und Produktionsmitteln in den Staatsbetrieben bei Beginn der Reformen auch keine wesentliche interne Verschuldung gegenüber dem privaten Sektor aus. Die Defizite des Staates ließen sich zunächst leicht verschmerzen, zumal China ja den Zinssatz für seine Staatsschulden ebenfalls kontrollierte, das heißt die Kosten der Staatsverschuldung weitgehend selbst bestimmen konnte. Des Weiteren konnte man mittels der Notenpresse die interne Inflation zum Zweck der finanziellen Repression gegen seine Bürger nutzen.

Trotzdem musste China aufgrund einer Vielzahl fauler Kredite, die von den staatlichen Geschäftsbanken in dieser Phase vergeben worden waren, seinen Geschäftsbanken in den Jahren 2003/2004 aus der Klemme helfen. ${ }^{68}$ Seither war die chine-

68 »China's Vice Minister of Finance Lou Jiwei said in Beijing that the Chinese government is prepared to inject money into the Industrial and Commercial Bank of China (ICBC) and the Agricultural Bank of China (ABC), two of the country's s big four s state owned banks «; Xinhua 2004. Zuvor waren die beiden anderen chinesischen staatlichen Geschäftsbanken Construction Bank und Bank of China mit einer Bürgschaft über 45

Leviathan, 42. Jg., 1/2014 
sische Staats- und Parteiführung sehr daran interessiert, sowohl das Management seiner Geschäftsbanken bei der Kreditvergabe als auch die Aufsicht und Kontrolle seitens der chinesischen Zentralbank deutlich zu verbessern. Entsprechend suchte man auch hier westliches Know-how: Partnerbanken im Westen sollten den chinesischen Bankern eine effizientere Kreditvergabepraxis beibringen. Leider hatte man in diesem Fall den Bock zum Gärtner gemacht.

\section{Die Große Wirtschafts- und Finanzkrise von 2008}

Die globale Finanzkrise des Jahres 2008 zeigte, dass die kapitalistischen Lehrmeister ebensolche Dilettanten waren wie ihre chinesischen Eleven. Das war eine wichtige Lektion für die chinesische Staatsführung, da der Nimbus der westlichen Überlegenheit einen kräftigen Dämpfer erhielt. Das financial engineering der großen westlichen Geschäftsbanken, angeführt von der Wall Street und der Londoner City, war keineswegs so effizient, wie man es den Chinesen hatte weismachen wollen. ${ }^{69}$ Das hat das Selbstbewusstsein Chinas gegenüber dem Westen enorm gestärkt.

Trotz dieser zwiespältigen Erfahrungen mit einer Politik der massiven Kreditvergabe und des deficit spending nach der Asienkrise war die chinesische Staatsführung sofort bereit, erneut ein gewaltiges Konjunkturpaket aufzulegen, ${ }^{70}$ als sich nach der Pleite von Lehman Brothers am 15. September 2008 ein weltweiter Konjunktureinbruch abzeichnete. ${ }^{71}$ China wollte unter allen Umständen verhindern, dass es auch im eigenen Lande zu einem drastischen Konjunktureinbruch wie in Deutschland oder Japan kommt. Laut Plan sollte ein jährliches Wirtschaftswachstum von mindestens 7,5 Prozent aufrechterhalten werden. Es sollten genügend Arbeitsplätze entstehen, um die Landflucht zu bewältigen und die soziale Stabilität des Landes nicht zu gefährden. Nach offiziellen Statistiken Chinas, die vom IWF regelmäßig im World Economic Outlook publiziert werden, stieg die Staatsschuldenquote von 18 Prozent des Bruttoinlandsprodukts im Jahr 2008 auf rund 34 Prozent im Jahr 2009 an. Mithin wurde ein zusätzliches Staatsdefizit von 16 Prozentpunkten im Jahr 2009 zur Konjunkturstützung in Kauf genommen. China übertraf damit die Staatsdefizite sämtlicher anderer Länder deutlich (das US-Konjunkturpaket betrug im Vergleich dazu nur 14 Prozent und das deutsche nur sieben Prozent). In diese Berechnung

Mrd. US-Dollar vom chinesischen Staat gerettet worden. Über diese vier großen staatlichen Geschäftsbanken (big four) wird der größte Teil des Kreditgeschäfts in China insbesondere auch mit den chinesischen Staatsbetrieben abgewickelt. Mithin hatten die vier Banken zuvor in erheblichen Umfang Kredite an Staatsbetriebe vergeben, die sich als faul entpuppten. Was sich in den USA und Europa als Skandal der Rettung privater Geschäftsbanken durch den Staat darstellt, ist in China eben nur ein innerstaatlicher Vorgang. Es zeigt aber an, dass es erhebliche Fehlinvestitionen in China seitens der Staatsbetriebe speziell nach der Asienkrise gegeben haben muss.

69 Das 2010.

$70 »$ China has, in particular, managed to keep their economy growing in 2009 at a rate of 8.7 per cent, which has supported by the massive stimulus package put together by the Chinese authorities (amounting to US\$ 585 billion) «; Verick, Islam 2010, S. 5.

71 Handelsblatt 2008. 
gingen jedoch nur die Schulden der chinesischen Zentralregierung ein, diejenigen der Provinzregierungen, der Kommunen, der Staatsbanken sowie der Staatsbetriebe blieben unberücksichtigt.

Üblicherweise ist in den westlichen Ländern die Zentralregierung zuständig für die Konjunktursteuerung und die Finanzierung zusätzlicher Staatsausgaben. In Deutschland soll nicht nur dem Bund, sondern auch den Bundesländern und den Kommunen gemäß der jetzt im Grundgesetz verankerten Schuldenbremse die autonome Aufnahme von Krediten zur Defizitfinanzierung ganz untersagt werden. In China stellt sich dies völlig anders dar: Hier spielen die Provinzregierungen und die Kommunen im Verband mit den Staatsbanken und den Staatsbetrieben eine wesentlich größere Rolle für die Konjunkturbelebung als die Zentralregierung. Zwar soll nach offiziellen Angaben die chinesische Staatsschuldenquote der Zentralregierung für das Jahr 2013/14 voraussichtlich auf nun wieder 20 Prozent sinken, ${ }^{72}$ aber das muss keineswegs bedeuten, dass in den anderen Bereichen des chinesischen Staates bereits eine Konsolidierung stattgefunden hat. China leistet sich eben, wenn es um die Staatsverschuldung geht, auch eine gewaltige Schuldenlast, die aber außerhalb des Etats der chinesischen Zentralregierung, nämlich in den genannten Bereichen der Staatswirtschaft, bilanziert wird. Wie bereits im Gefolge der Asienkrise und der damals betriebenen Konjunktursteuerung der chinesischen Regierung festgestellt werden konnte, wurden dabei in großem Umfang Kredite von den chinesischen Staatsbanken gewährt, die sich später als faule Kredite (non-performing loans, NPLs) herausstellten.

Die insbesondere seit seinem Beitritt zur Welthandelsorganisation im Jahr 2001 gewachsene Integration Chinas in die Weltwirtschaft hatte eben auch eine Schattenseite. Da China seit Langem von den USA gedrängt wurde, sein exportorientiertes Wirtschaftswachstum mehr auf die chinesische Binnennachfrage, insbesondere den privaten Konsum auszurichten, bot sich hier durch die Rezession in den Exportmärkten Europas und der USA die Chance einer Korrektur des chinesischen Wachstumsmodells. Die ehemalige sozialistische Planwirtschaft oder nun keynesianisch gesteuerte Marktwirtschaft mit chinesischen Merkmalen drohte aufgrund ihrer hohen Exportquote durch die Weltkonjunktur destabilisiert zu werden. Denn der Anteil ausländischer Unternehmen, die China als Plattform für den Export in die Märkte in den USA und Europa benutzen, ist hoch, und die Gefahr bestand, dass diese schneller bereit sein würden, ihre Produktionen und die ihrer Subunternehmer in China zurückzufahren, weil sie so unter dem Druck der Öffentlichkeit die eigenen Produktionsstandorte in ihren Heimatländern in Europa und in den USA schonen und die Anpassungslasten nach China externalisieren können. China wurde daher von der Nachfrageschwäche in den USA und Westeuropa sogar härter getroffen als die Wirtschaft in den USA und Europa.

Lag die chinesische Exportquote im Jahr 2000, das heißt vor dem Beitritt zur WHO, im Verhältnis zum Bruttoinlandsprodukt nach offiziellen Angaben der Weltbank bei 23 Prozent, so stieg sie bis zum Jahr 2011 auf 31 Prozent an. Darin sind die über Hong Kong und Macao exportierten Waren und Dienstleistungen nicht

72 IWF 2013, S. 5.

Leviathan, 42. Jg., 1/2014 
enthalten, ${ }^{73}$ was darauf hindeutet, dass dies noch eine eher konservative Schätzung der Abhängigkeit Chinas vom Weltmarkt ist. Im internationalen Vergleich sollte Chinas Exportquote nicht viel mehr als fünf Prozent betragen, wenn man die Größe des Landes und seines Binnenmarktes als Maßstab heranzieht. Im Vergleich zur Mao-Ära ist China durch exogene Schocks der Weltwirtschaft daher jetzt viel verletzbarer geworden. Wenn Konjunkturstörungen zuvor durch die chinesische Kommandowirtschaft in der Mao-Ära mit entsprechenden harten internen Schocks bewältigt wurden, konnten solche wirtschaftlichen Einbrüche jetzt sanfter mit einer keynesianischen Politik der Stützung der Nachfrage kompensiert werden. Dabei fand auch in China die Konjunkturstützung seitens des Staates auf Pump statt, das heißt die zusätzlichen Ausgaben des Staates wurden nicht aufkommensneutral finanziert. Es stellt sich nun ebenso wie in den kapitalistischen Ländern des Westens ein Staatsschuldenproblem, das langfristig angegangen werden muss. Allerdings werden die Schulden gegenüber der eigenen Bevölkerung gemacht, das heißt deren Ersparnisse werden in zusätzliche Staatsausgaben umgeleitet, sodass sich daraus keine Zahlungsbilanzstörungen ergeben.

Fraglich ist, ob die vom IWF für China veröffentliche Staatsschuldenquote das chinesische Staatschuldenproblem adäquat widerspiegelt. In einer umfangreichen Studie auf der Grundlage öffentlich zugänglicher Daten über die chinesischen Finanzstatistiken aller zuvor genannten Körperschaften kommen Walter und Howie zum Ergebnis, dass unter Einbeziehung insbesondere der vier großen Staatsbanken die Staatverschuldungsquote, gemessen am Bruttoinlandsprodukt im Jahr 2012, vermutlich bei 39 Prozent gelegen hat. ${ }^{74}$ Berücksichtigt man darüber hinaus noch die Verschuldung der Provinzen und Kommunen, so liegt man bereits bei 69,7 Prozent. Da es innerhalb des Systems der chinesischen Staatsfinanzen jedoch aufgrund von nicht erfolgten Wertberichtigungen von NPLs an Staatsbetriebe, Provinzregierungen und Kommunen einen Korrekturbedarf von rund 12,1 Prozent des Bruttoinlandsprodukts gab, läge nach einer solchen Schätzung die chinesische Staatsschuldenquote, gemessen am BIP, bei 81,8 Prozent. Damit wäre sie mit den für Deutschland offiziell angegebenen 81,9 Prozent durchaus vergleichbar. China könnte daher weitaus weniger solide dastehen, als im Allgemeinen angenommen wird, zumal wenn die Gefahren der Schuldendynamik von der Regierung nicht eingedämmt werden. ${ }^{75}$

73 Siehe http://wdi.worldbank.org/table/4.8 (Zugriff vom 19.12.2013).

74 Walter, Howie 2012, S. 221-222.

$75 \mathrm{Zu}$ dem deficit spending kam jedoch noch eine gewaltige Kreditexpansion über das chinesische Banken- und Schattenbankensystem hinzu. » [...] in 2009 and 2010 thing went too far. Spurred on by the government, China's banks increased their lending by almost 9.6 trillion Yuan ( $\$ 1.5$ trillion) in 2009. That is roughly twice the size of the Indian banking system, as Bank Credit Analyst, a research company, has pointed out. In other words, China's lenders added two Indian to their loan book in the space of a year "; Cox 2011. Zu Chinas Schattenbanken, das heißt privaten Kreditgebern außerhalb der Kontrolle des Staates, siehe auch Erber 2013 b. 
Abbildung 3: Leitzinssatz der chinesischen Zentralbank (PBC), 2005-2013

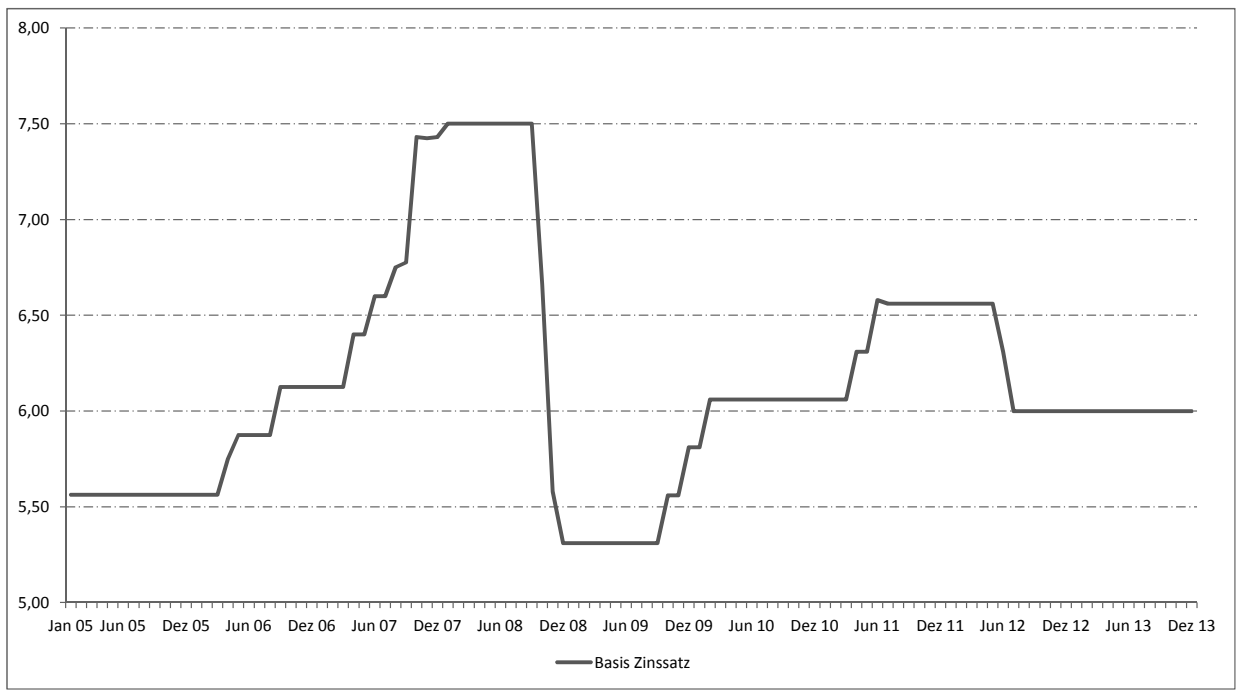

Quelle: PBC.

\section{Chinas Wachstumsbremse 2013}

Bereits 2013 wurde erneut ein Konjunkturpaket aufgelegt, um die zuletzt schwächelnde Wirtschaft weiter zu beleben. Dabei stehen der Ausbau der Eisenbahninfrastruktur, der bisher von der chinesischen Staatsbahn betrieben wurde und jetzt für private Investoren geöffnet werden soll, sowie verbesserte Finanzierungsmöglichkeiten für kleine und mittlere chinesische Unternehmen (KMUs) durch den offiziellen Bankensektor im Vordergrund. Auch Steuererleichterungen und die Streichung der Mehrwertsteuer von 7,5 Prozent sind vorgesehen. ${ }^{76}$ Damit hofft man das Wirtschaftswachstum auf 7,5 Prozent in diesem Jahr stabilisieren zu können.

Parallel dazu wurde die Kreditvergabe an den privaten Sektor und insbesondere die privaten Haushalte erneut gelockert, sodass insbesondere die Kredite für den Immobilienerwerb ${ }^{77}$ und später für den PKW-Kauf ausgeweitet werden konnten.

\subsection{Nachhaltig lockere chinesische Geldpolitik}

Durch eine Niedrigzinspolitik der chinesischen Zentralbank - gemessen an chinesischen Verhältnissen - wurde so die Liquidität auch in China durch Kreditgeldschöpfung massiv ausgeweitet (Abbildung 3).

China droht dabei nicht wie den westlichen Ländern eine Liquiditätsfalle durch Rezession infolge Nachfrageschwäche und schwacher Ertragsaussichten, sodass

76 Geinitz 2013; Reuters 2013.

77 Rabinovitch 2013.

Leviathan, 42. Jg., 1/2014 
Abbildung 4: Veränderungen des Verbraucherpreisindex in China, 1994-2013

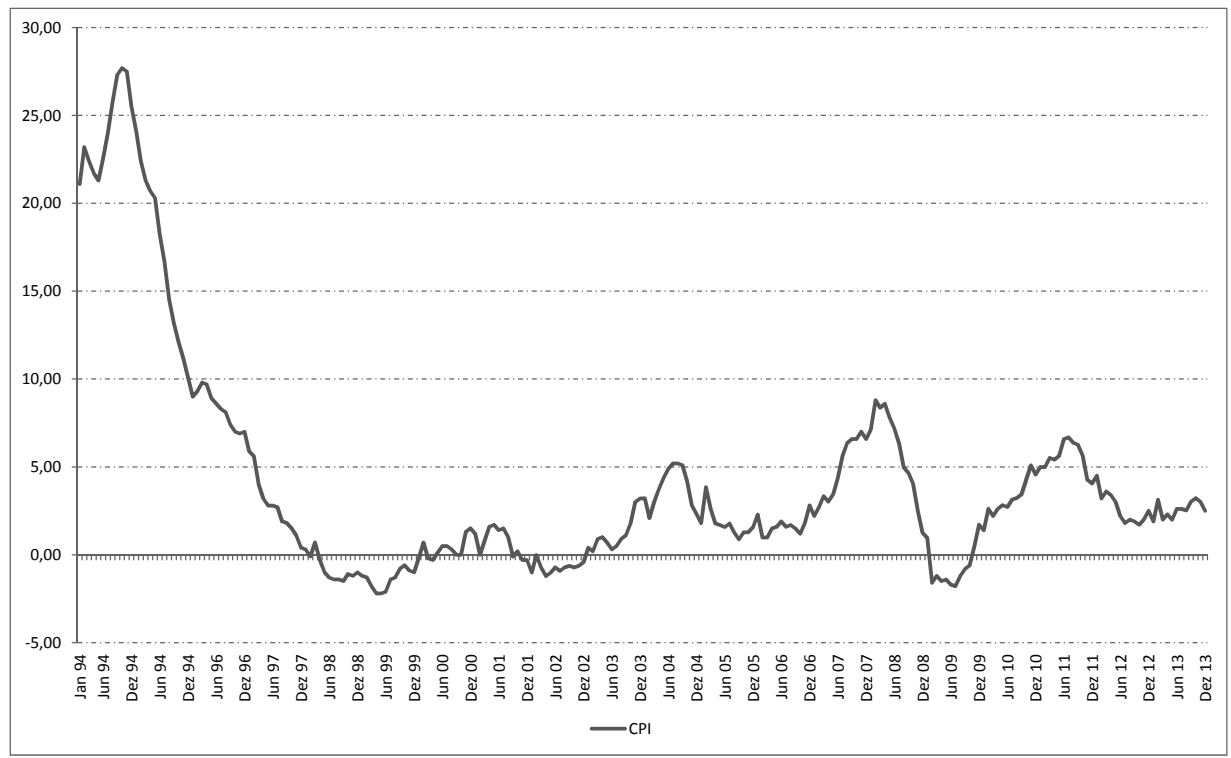

Quelle: PBC.

man dort auf unorthodoxe Geldpolitik à la Bernanke verzichten kann, weil noch genügend attraktive und gewinnbringende Anlagemöglichkeiten vorhanden sind. Aufgrund der hohen Wachstumsrate der chinesischen Wirtschaft von mehr als 7,5 Prozent wirkte diese lockere Geldpolitik eines niedrigen Leitzinses über die gesamte Zeitspanne der zurückliegenden Jahre seit 2005 permanent expansiv. Vielleicht einfacher: Im Widerspruch zu Wicksells ${ }^{78}$ Gleichgewichtsbedingung hält China den Realzins permanent unter der Wachstumsrate der Wirtschaft. Zwar kann aufgrund hoher Inflation in China der Realzins negativ sein, aber der Nominalzins bleibt im positiven Bereich oberhalb von fünf Prozent.

Da die Regierung direkt auf die Kreditvergabe der staatlichen chinesischen Geschäftsbanken Einfluss nimmt, kann sie durch Einschränken der Kreditvergabe dieser Institute Konjunktursteuerung betreiben. Nicht der Kreditzins, sondern der Zugang zu Krediten ist dabei die eigentlich bindende Restriktion in China. Wegen der periodisch hohen Inflationsraten führt das teilweise sogar zu negativen Realzinsen, wenn die Inflationsrate das Wachstum auffrisst (Abbildung 4).

Das senkt zwangsläufig wegen der damit verbundenen finanziellen Repression die Bereitschaft, Geldvermögen zu halten. Stattdessen wird Realbesitz als die bessere Option der Wertsicherung betrachtet, wenn nicht sogar die stille Kapitalflucht ins Ausland über das im Folgenden beschriebene Schattenbankensystem gewählt wird

78 Wicksell 2005 [1898]. 
als Möglichkeit, Geldvermögen im Ausland in Fremdwährungen zu halten. Solange der Wechselkurs des Renminbi zum US-Dollar stabil gehalten wurde, gab es kein Wechselkursrisiko. Das hat sich nun seit einigen Jahren durch die Aufwertung des Renminbi zum US-Dollar und anderen Weltreservewährungen geändert: Wer die Renminbizone verlässt, kann entsprechend auch Verluste durch den Wechselkurs erfahren.

\subsection{Chinesisches Schattenbankensystem}

Neben den staatlichen Geschäftsbanken gibt es in China ein privates Schattenbankensystem. Da der Zugang zu den staatlichen Geschäftsbanken insbesondere für private kleine und mittlere Unternehmen in der Regel schwierig ist, hat sich ein solches Parallelsystem entwickelt, durch das sie sich finanzieren können. Analog zur Korruption, die als »Schmiermittel « Geschäftsprozesse zu beschleunigen hilft, füllte das chinesische Schattenbankensystem also eine Marktlücke im chinesischen Finanzwesen. Die Effizienz der chinesischen Finanzaufsicht über die Geld- und Kapitalmärkte leidet jetzt - nicht anders als im Westen - an der Intransparenz des Finanzsektors: Es gibt keine ausreichenden Informationen über die mit dem chinesischen Schattenbankensystem verbundenen Risiken. Mit der sich derzeit abzeichnenden Abschwächung der Wirtschaftsentwicklung in China steigen insbesondere auch hier die Kreditausfallrisiken. ${ }^{79}$

Da dieser Teil der chinesischen Wirtschaft über die Jahre ein immer größeres Gewicht an der chinesischen Volkswirtschaft eingenommen hat - Schätzungen gehen von einer Größenordnung des Schattenbankensystems von 5,8 Billionen USDollar aus,$-{ }^{80}$ reduziert das auch die Wirksamkeit der chinesischen Geld- und Kreditpolitik in seiner bisherigen Form. Verglichen mit der Preisstabilität in den USA und Europa, sind die Inflationsraten in China hoch volatil. Die seit Frühjahr 2013 regierende neue Staatsführung unter Xi Jingping und Li Keqiang hat als eine erste Maßnahme dem Schattenbankensystem den Kampf angesagt. Das hat vorübergehend zu einer massiven Liquiditätsklemme geführt, ${ }^{81}$ die inzwischen jedoch behoben scheint und einer Konsolidierung der Kreditexpansion Platz gemacht hat. ${ }^{82}$ Das verbleibende Problem ist das der Überhitzung des Marktes für Immobilien, die von vielen als Realvermögensanlage gewählt worden waren. Diesem Problem konnte bisher noch nicht wirkungsvoll entgegengewirkt werden, ${ }^{83}$ was seine Ursache darin haben kann, dass es immer noch Mittel und Wege gibt, sich der Kontrolle durch die Geldpolitik zu entziehen.

79 »Call it the new China Syndrome: Although Asia's biggest economy is slowing down markedly, credit continues to surge. Dead-end projects and dying industries are sucking up an ever-larger portion of new credit, while more productive borrowers are starved for funds «; Wildau 2013.

80 Roberts 2013 a.

81 Hille 2013.

82 Bloomberg 2013.

83 CNNMoney 2013.

Leviathan, 42. Jg., 1/2014 
Eine wichtige Rolle spielt beim Umgehen der geldpolitischen Kontrolle der Kapitalzufluss und Kapitalabfluss durch Manipulationen von Außenhandelsrechnungen. Um Gelder ins Ausland zu transferieren, ${ }^{84}$ werden je nach Bedarf zu hohe oder zu niedrige Rechnungen bei Importen und Exporten - gemessen an üblichen Weltmarktpreisen - ausgewiesen, während die realen Preise bezahlt werden. Auf diesem Weg lassen sich Gelder von und nach China an den staatlichen Aufsichtsbehörden vorbeischleusen. Hinzu kommt ein intensiver Schmuggel von Waren, die falsch deklariert und deshalb mit zu niedrigen Zollsätzen belegt werden. ${ }^{85}$ Gewinne aus solchen Geschäften sind es, die dann als Finanzierungsgrundlage für das chinesische Schattenbankensystem Verwendung finden. Die chinesischen Kapitalverkehrskontrollen werden vermutlich in erheblichem Umfang insbesondere im Privatsektor unterlaufen. ${ }^{86}$ Das schafft für die chinesische Geldpolitik das bekannte Trilemma, dass man die zwei Zielgrößen - inländische Preisstabilität und feste Wechselkurse bei quasi-offenen Kapitalmärkten - nicht gleichzeitig kontrollieren kann. Umgekehrt könnte dies aber auch die möglicherweise bevorstehende offizielle Aufhebung der Kapitalverkehrskontrollen erleichtern, weil diese bereits jetzt nur scheinbar wirksam beziehungsweise zu einem erheblichen Teil unwirksam sind.

In Bezug auf den Kapitalverkehr spielt Hong Kong als eigenständige Einheit mit eigenen Zollgesetzen eine Schlüsselrolle. Hong Kong ist quasi ein Finanzplatz außerhalb der Grenzen und doch innerhalb Chinas mit einer eigenen Währung und Jurisdiktion. Durch die Bindung des Hong Kong-Dollar an den US-Dollar und sein international engvernetztes Bankensystem mit Zweigstellen aller großen chinesischen Staatsbanken eignet sich Hong Kong besonders gut für jedwede Art von Geldwäscheoperationen von und nach China und insbesondere mit Taiwan und den USA. Daneben nimmt auch das vorwiegend von Chinesen bevölkerte Singapur für den ASEAN-Raum eine wichtige Rolle bei der Verflechtung des Schattenbankensystems Chinas mit der übrigen Welt ein. Ebenso wie der westlichen Finanzaufsicht ist den chinesischen Beamten die Kontrolle über diese spezifischen Schattenbankensysteme weitgehend entglitten, was zuletzt dazu geführt hat, dass man sich auf dem G20-Gipfel in St. Petersburg auf einen Fahrplan zu einer internationalen Kontrolle des weltweiten Schattenbankensystems verständigen konnte. ${ }^{87}$ Zahlreiche private chinesische multinationale Unternehmen haben seither ihren Firmensitz nach Hong Kong verlegt. Gelänge es, das Schattenbankensystem Chinas auszutrocknen, wäre für eine Liberalisierung der Kapitalmärkte und eine Freigabe des Wechselkurses des Renminbi ein wichtiger Stolperstein aus dem Weg geräumt. ${ }^{88}$ Hong Kong bietet bereits jetzt die Möglichkeit, Renminbi-Konten und Geldgeschäfte wie Bonds und Aktienhandel neben denen mit anderen ausländischen Währungen abzuwickeln. Es wurde so zum führenden Finanzplatz, der als Bestandteil einer zukünftigen Ren-

84 Xinhua 2011.

85 Fisman, Miguel 2008.

86 PriceWaterhouseCoopers 2013.

87 Dohms 2013.

88 Fung 2013. 
minbi-Zone eine zentrale Rolle einnimmt. Nun soll auch Schanghai eine Freihandelszone erhalten, die ausländischem Kapital analog zur Situation in Hongkong besondere Freizügigkeit bieten soll. ${ }^{89}$

Angesichts einer blühenden Korruption in Partei und Staat dürfen wir davon ausgehen, dass die Grenzen zwischen Schattenbankensystem und offiziellen Banken fließend sind. Letztendlich wird auch hier - wie in der westlichen Welt - die Frage, ob eine striktere Regulierung der Finanzmärkte in China erfolgreich sein wird, erst in der Zukunft beantwortet werden können.

\subsection{Hayeks Malinvestments in China}

Aus Hayek'scher Sicht ergeben sich in China Probleme hinsichtlich der durch zu niedrige Zinssätze angeregten Fehlinvestitionen (Malinvestments). ${ }^{90}$ China hat einen hohen Investitionsbedarf bei der Infrastruktur, um seinen Wachstumspfad beibehalten zu können. ${ }^{91}$ Entsprechend der Aschauer-Hypothese ${ }^{92}$ könnte man unterstellen, dass jeder Yuan ${ }^{93}$, der in den Ausbau der chinesischen Infrastruktur investiert wird, langfristig positive Erträge durch höhere Produktivität und höheres gesamtwirtschaftliches Wachstum zeigen wird. Die extrem hohen Investitionsquoten in China haben jedoch zahlreichen empirischen Analysen zufolge das Problem, dass aufgrund der günstigen Finanzierungskonditionen, die insbesondere chinesischen Staatsbetrieben und Provinzregierungen und Kommunalverwaltungen eingeräumt werden, nicht in die für das Wachstum nötige Infrastruktur, sondern eher in prestigeträchtige Projekte investiert wird, die aus ökonomischer Sicht unsinnig und unrentabel sind. Ein extremes Beispiel hierfür ist New Ordos, eine Geisterstadt in der Inneren Mongolei. »Diese wurde für eine Einwohnerzahl von rund 1 Million Menschen errichtet. Schuld haben die Kohle und das Gas. Als man im Jahr 2000 südlich von Dongsheng gewaltige Vorkommen entdeckte - Schätzungen zufolge um 15 Prozent der gesamten Kohle- und 30 Prozent der gesamten Erdgasreserven Chinas -, erweiterte man die Stadtgrenzen, nannte die Stadt auf einmal Ordos und schuf New Ordos, ein Wohnviertel für eine Million Menschen. «94 Im Jahr 2011 wohnten dort jedoch nur etwa 5.000 Menschen. Eine gewaltige Fehlinvestition.

Die Kommunen spielen eine entscheidende Rolle bei der Privatisierung von Land, das zuvor in Staatsbesitz war. Mit den Privatisierungserlösen werden dann in der Regel Kommunalprojekte finanziert. Darüber hinaus bietet sich bei Geschäften der Kommunen reichlich Gelegenheit für Korruption und Bestechung. Man kann sich

89 Rimmele 2013.

90 Cox 2012.

91 Isaksson 2010.

92 Aschauer 1989.

93 »Renminbi« (bedeutet Volkswährung) ist die offizielle Bezeichnung der chinesischen Währung, während der Begriff »Yuan « (bedeutet Ur-Anfang) die Grundeinheit der chinesischen Währung bezeichnet. Oftmals werden sie umgangssprachlich synonym verwendet.

94 Der Spiegel 2012.

Leviathan, 42. Jg., 1/2014 
durch günstige Preissetzung beim Verkauf von Staatsland entsprechende Zahlungen vom Landkäufer zusichern lassen. Ebenfalls kann sich die Partei- und Staatsbürokratie an der Durchführung solcher Großprojekte durch Beteiligungen und Beraterverträge mit der Bauindustrie illegal bereichern. Das Beispiel des chinesischen Vize-Eisenbahnministers macht deutlich, wie große Infrastrukturprojekte bei der Vergabe bereits mit stillen Bestechungszahlungen an Entscheidungsträger belastet werden können. Darüber hinaus können durch mangelhafte Aufsicht und Kontrolle bei der Ausführung von Bauprojekten massive Schäden entstehen. ${ }^{95}$

»Ein altes Beratersprichwort in China besagt: Es ist gut, die geschriebenen und veröffentlichten Gesetze zu kennen. Noch besser ist es, auch die nicht veröffentlichten Gesetze zu kennen. Am besten ist es jedoch, wenn man auch die ungeschriebenen Gesetze kennt und den Grundsatz beherrscht: `China ist groß und der Kaiser lebt in Beijing « "96 Auf dieser Grundlage können Projekte, die dem Anspruch nach der Wirtschaftsstruktur und damit der Effizienz der chinesischen Wirtschaft dienen sollen, rasch eine gegenteilige Wirkung entfalten. Fehlkalkulationen gewaltigen Ausmaßes, mangelhafte Funktionstüchtigkeit der erstellten Bauten etc. gehören zum Alltag chinesischer Bauprojekte, da die Entwicklung rascher voranschreitet als die Ausbildung entsprechender Fachkompetenz und Kontrollkompetenz.

Das Problem ist also nicht vorrangig die makroökonomische Zuweisung von großen Finanzbeträgen zur Infrastrukturentwicklung, sondern deren praktische Umsetzung, sodass unter Effizienzgesichtspunkten auch die chinesische Wirtschaft davon profitiert. Mangelnder Wettbewerb und fehlende Kontrolle haben am Ende adverse Effekte. Da es jedoch an öffentlichen Debatten sowie kritischer Berichterstattung in China fehlt und Versuche, dies im Internet in China stattfinden zu lassen, von der Regierung rigoros unterbunden werden, haben sich in weiten Teilen der chinesischen Wirtschaft im Zuge der erlaubten Public Private Partnership (PPP) Strukturen entwickelt, die die Vorgaben der chinesischen Zentralregierung ins Leere laufen lassen. Werden solche Projekte dann von den staatlichen Geschäftsbanken finanziert, dann kommt es in erheblichem Umfang zu den bereits zuvor erwähnten NPLs.

\section{Zwischenfazit}

China hat sich ohne große Probleme Keynes' Prinzipien der makroökonomischen Konjunktursteuerung angeeignet, aber es ergibt sich das Problem, dass die aufgelegten Konjunkturprogramme trotz der hohen wirtschaftlichen Wachstumsrate aufgrund systemischer Ineffizienzen die Staatsdefizite ansteigen lassen, ohne durch entsprechende Erträge und ihre Multiplikatoreffekte wieder ausgeglichen zu werden.

95 Ai Weiwei, der inzwischen weltberühmte chinesische Künstler, hat den Pfusch am Bau von öffentlichen Schulgebäuden in Sichuan angeprangert. Beim Erdbeben im Jahr 2008 wurden dabei tausende von Schulkindern getötet. Siehe www.youtube.com/watch? v=PodFm4qbADw (Zugriff vom 19.12.2013).

96 Siehe www.chinaproject.de/Asien-Pacific/privatisierung_in_china.htm (Zugriff vom 19.12.2013). 
Auch in China droht das von Reinhard und Rogoff ${ }^{97}$ konstatierte Problem, dass eine kumulativ wachsende Staatsverschuldung das Wirtschaftswachstum aufgrund hoher Zinslasten und unzureichender Erträge deutlich abschwächen wird. Im Sinne von Hayeks monetärer Konjunkturtheorie drohen die starken Zinsschwankungen China in einem Boom-und-Bust-Zyklus gefangen zu halten. ${ }^{98}$ Durch den wachsenden Kontrollverlust über große Teile der chinesischen Wirtschaft wird die Zentralregierung in China vor neue Herausforderungen gestellt, die zwar denen in den westlichen Demokratien ähneln, aber doch besonderer Art sind, denn ihr fehlt das Korrektiv der Binnenlegitimation durch Parlamentarismus und unabhängiger Gerichtsbarkeit. Man ist daher in der Regel gezwungen, die Probleme hinter verschlossenen Türen zu lösen. Das untergräbt jedoch das Vertrauen der chinesischen Bevölkerung, da Gerüchte konkrete Informationen ersetzen und durch die digitalen Medien trotz aller Überwachungsversuche mittels der Großen Chinesischen Firewall ${ }^{99}$ verbreitet werden.

\section{Schlussfolgerungen}

China hat trotz seines rasanten wirtschaftlichen Aufstiegs in der Weltwirtschaft gegenwärtig mit erheblichen strukturellen Defiziten zu kämpfen. Das bisher so erfolgreiche Entwicklungsmodell einer exportorientierten Entwicklung, gestützt auf massiven Import von Wissen und Technologie durch die Ansiedlung westlicher und japanischer multinationaler Konzerne, stößt derzeit an seine Grenzen durch den wachsenden Widerstand sowohl der einzelnen Länder wie auch der daran beteiligten Unternehmen. Es besteht zudem trotz des Beitritts Chinas zur WHO die Gefahr wachsender Konflikte mit den USA und der EU sowie von Handelssanktionen.

Die bisherigen komparativen Vorteile - niedrige Lohnkosten und ein scheinbar unerschöpfliches Arbeitskräfteangebot - gehen nicht zuletzt durch die Folgen der Ein-Kind-Politik langsam verloren. Die jetzige, zahlenmäßig kleinere junge Generation entwickelt höhere Ansprüche an ihren zukünftigen Lebensstandard. Dies schließt nicht nur materiellen Wohlstand ein, sondern auch ein größeres Maß individueller Freiheit. Die gesellschaftliche Stabilität Chinas hängt daher sehr stark davon ab, ob es der politischen Führung gelingt, diesen Ansprüchen insbesondere der neuen Mittelschicht gerecht zu werden.

97 Reinhart, Rogoff 2010.

98 Schularick, Taylor 2009.

99 Kolonk 2013; Siemons 2013; Pilling 2013. »Das Projekt Goldener Schild, manchmal auch als die Große Firewall von China in Anlehnung an die chinesische Mauer (englisch Great Wall of China) bezeichnet, ist ein Projekt des Ministeriums für Staatssicherheit (MfS) zur Überwachung und Zensur des Internetverkehrs in China. Die Entwicklung wurde im Jahr 1998 gestartet und das Projekt 2003 landesweit in Betrieb genommen. Dem MfS zufolge stellt das Projekt ein Kommunikations- und Informationsnetzwerk dar, welches das Potenzial und die Effizienz der Exekutive steigern soll «; http://de.wikipedia .org/wiki/Projekt_Goldener_Schild (Zugriff vom 19.12.2013).

Leviathan, 42. Jg., 1/2014 
China konnte durch die bisher dominierende Rolle von Partei und Staat die chinesische Wirtschaftsentwicklung günstig gestalten. Aber aufgrund der traditionellen Strukturen ist mit dem rasanten Wirtschaftswachstum auch die Korruption in Partei und Staat auf ein vorher ungekanntes Ausmaß angewachsen. Das bedroht zunehmend die Legitimität der chinesischen Kommunistischen Partei und Staatsführung. Wen Jiabao, der frühere chinesische Premierminister, hat es auf den Punkt gebracht: Chinas Wirtschaft ist »instabil, unausgewogen, unkoordiniert und nicht nachhaltig. «100 Viele der Fragilitäten der chinesischen Wirtschaft ergeben sich aus dem komplexen Zusammenspiel zwischen dem Staat in engerem Sinne, den Parteimitgliedern, den staatlichen Geschäftsbanken und den Staatsbetrieben. Die Durchdringung mit Korruption auf allen Ebenen macht die Umsetzung politischer Ziele zunehmend schwieriger.

Der private Sektor entwickelt parallel dazu ein Eigenleben. Auch hier herrschen Korruption und Vetternwirtschaft. Ein weitgehend unkontrollierbares Schattenbankensystem macht die Kontrolle über die Kreditgeldschöpfung immer schwieriger, zumal über die vielen außenwirtschaftlichen Beziehungen durch vielfältige Formen des falschen Deklarierens (transfer pricing) und andere illegale Methoden die offiziellen Kapitalverkehrskontrollen mit dem Ausland unterlaufen werden.

Das Ziel, China zu einer der führenden Weltmächte aufsteigen zu lassen, birgt typische Risiken: ${ }^{101}$ China hat eine lange Tradition der strategischen Überdehnung, die insbesondere auch die Ming-Dynastie 1644 am Ende hat scheitern lassen. Die überambitionierten Projekte (Chinesische Mauer, Kaiserkanal, gewaltige Flottenexpedition von Zheng He, Verlegung der Hauptstadt von Nanjing nach Beijing und Bau der Verbotenen Stadt) und zahlreiche kriegerische Auseinandersetzungen verschlangen gewaltige Ressourcen und belasteten die chinesische Landbevölkerung, die die Hauptlast zur Staatsfinanzierung beizutragen hatte. Die permanente Finanzkrise und innere Unruhen erodierten den chinesischen Staat. Aufgrund der inneren Zerrüttung fiel es den Mandschu am Ende leicht, die Ming-Dynastie zu stürzen, da sie die Legitimation bereits bei der eigenen Bevölkerung verloren hatte. ${ }^{102}$

Chinas derzeitiger Führung droht ebenfalls eine strategische Überdehnung. Man will innerhalb sehr kurzer Zeit zu einer unangefochtenen Weltmacht aufsteigen. Das erfordert gewaltige Ressourcen und finanzielle Opfer für den Aufbau einer industriellen Infrastruktur und zugleich einer Rüstungsindustrie. Um dieses Ziel verwirklichen zu können, hat China den Weg einer keynesianischen Politik der Nachfragesteuerung eingeschlagen, ${ }^{103}$ die immer, wenn das exportgetriebene Wachstum in China zu erlahmen droht, dieses durch staatliche Ausgabenprogramme kurzfristig stimulieren soll. Allerdings bilden sich dabei stille Lasten in Form einer steigenden Staatsverschuldung. Gleichzeitig wird aber bisher der chinesische Mittelstand gebremst und werden die chinesischen Staatskonzerne bevorzugt, die keineswegs

$100 »[\ldots]$ unstable, unbalanced, uncoordinated, and unsustainable«; Bloomberg 2010.

101 Kennedy 2010.

102 Siehe http://de.wikipedia.org/wiki/Ming-Dynastie (Zugriff vom 19.12.2103).

103 Vgl. dazu Zinn 2013. 
besonders effizient wirtschaften. ${ }^{104}$ Sie haben jedoch in China den Status des $" z u$ groß, um zu scheitern " und können sich der finanziellen Unterstützung oder gar einer Rettung durch den Staat sicher sein. ${ }^{105}$

Durch eine Vielzahl von offenen und verdeckten Subventionen entstehen auf diese Weise massive Wettbewerbsverzerrungen. China hat somit nur halbherzige Schritte zu einer echten Marktwirtschaft vollzogen, die bereits seit einiger Zeit wieder zurückgenommen werden. Ob es unter diesen Umständen der neuen chinesischen Führung gelingt, den Supertanker China von seinem bisherigen Kurs abzubringen und die von zahlreichen Experten vorgeschlagenen umfassenden Strukturreformen - insbesondere auch der Weltbank oder der OECD - umzusetzen, bleibt völlig offen. Analog zu den japanischen Abenomics, mit der der japanische Ministerpräsident Shinzo Abe die seit Jahren vor sich hindümpelnde japanische Wirtschaft wieder auf einen Pfad höheren Wachstums führen will, wird auch China gewaltige interne Konflikte beim Auf- und Ausbau einer effizienten Verwaltung und Bürokratie bewältigen müssen, die gegebenenfalls die Initiative der chinesischen Bevölkerung zu einer umfassenden Modernisierung entfesseln kann. Das Beispiel Japans zeigt, dass es auch nach Jahrzehnten eines extrem hohen Wirtschaftswachstums ${ }^{106}$ durchaus zu einer massiven Wachstumskrise kommen kann. China ist davor nicht gefeit, wiewohl es bisher zahlreiche Beobachter gibt, die an den unvermeidlichen Aufstieg Chinas im 21. Jahrhundert glauben.

China könnte von der im Westen geführten heftigen Debatte Keynes versus Hayek durchaus profitieren, wenn man die richtigen Lehren daraus zieht. Es geht hier meiner Ansicht nach gar nicht um ein ausschließliches Entweder-oder, sondern um ein Sowohl-als-auch. Keynesianische Makrosteuerung kann in Zeiten der Krise ein wichtiges Instrument der Wirtschaftspolitik sein, um die Wirtschaft schneller wieder auf einen normalen Wachstumspfad zurückzuführen. Allerdings erfordert dies mehr als nur die Definition eines möglichst umfangreichen Finanzrahmens, der durch steigende Staatsverschuldung finanziert wird. Der Teufel steckt im Detail. In der praktischen Implementierung von Konjunkturprogrammen sind - die langjährigen Erfahrungen zeigen es - immer wieder gravierende Fehler begangen worden.

Als Rezept für die Überwindung einer schweren Strukturkrise ist eine solche Kreditpolitik nur bedingt geeignet, bestenfalls ein Schmerzmittel für die parallel dazu erforderlichen Strukturreformen. Ansonsten droht die chinesische Führung auf eine Krise der Staatsfinanzen zuzusteuern. Hat man sich in China möglicherweise die falschen Lehrmeister ausgesucht? Die USA scheinen derzeit noch immer nicht geneigt, sich den Herausforderungen einer grundlegenden Runderneuerung ihrer

\section{Roberts 2013 b.}

105 »In contrast, China's state-owned firms typically enjoy preferential treatment in terms of administrative approval and bank loans. Although most firms report profits, totalling US\$ 920 billion from 2001 to 2009, the state is saddled with a negative 1.47 per cent real average return on equity after accounting for US\$ 1.19 trillion of subsidy and foregone costs «; Yajing, Jie 2013.

106 Siehe Maddison Datenbank, www.ggdc.net/maddison/maddison-project/home.htm (Zugriff vom 19.12.2013). 
Wirtschaft zu stellen, wiewohl Obama sichtlich bemüht ist, die heimatliche Produktion zu fördern, um die Exportrate zu steigern. Aber die massiven Staatsdefizite wachsen weiter, und die Geldpolitik wird als Heilmittel eingesetzt. Auch China könnte sich in eine solche problematische Entwicklung verstricken. Es fehlt insbesondere an einer kritischen Öffentlichkeit und demokratischen Kontrolle, die Korrekturen rechtzeitig erzwingt.

Am Ende könnte es dann trotz der Reformpropaganda auch in China wieder lauten: Same, same, but different. Grundlegende Reformen können nicht durchgesetzt werden. Es gilt die alte Weisheit: Der Erfolg von gestern ist kein Garant für den Erfolg von morgen. China sollte sich dabei an Keynes Empfehlungen zur Stabilisierung der konjunkturellen Entwicklung halten und sich gleichzeitig den von Hayek benannten Gefahren von Konjunkturprogrammen in Form von Fehlinvestitionen und fehlenden Strukturreformen stellen. Nur wer sich rechtzeitig mit den binnenwirtschaftlichen Problemlagen auseinandersetzt und sich den globalen Veränderungen der Weltwirtschaft anpasst, kann auch weiter auf der Erfolgsspur bleiben.

\section{Literatur}

Aschauer, David A. 1989. »Is public expenditure productive? «, in Journal of Monetary Economics 23, 2, S. 177-200.

Automobilproduktion 2013. "VW eröffnet neues Motorenwerk in China ", in Automobilproduktion vom 17. August 2013.

Balassa, Bela 1981. The newly industrializing countries in the world economy. New York: Pergamon.

Bellow, Adam 2003. In praise of nepotism. A natural history. New York: Doubleday.

Bloomberg 2010. "China's Wen blocked by politics from fixing sunstable< economy «, in Bloomberg News vom 3. März 2010.

Bloomberg 2013. "China's credit expansion slows as Li curbs shadow banking ", in Bloomberg News vom 9. August 2013.

Boston Consulting Group 2011. Made in the USA, again: manufacturing is expected to return to America as China's rising labor costs erase most savings from offshoring. Press release. www.bcg.com/media/PressReleaseDetails.aspx?id=tcm:12-75973 (Zugriff vom 19.12.2013).

China Briefing 2013. Why corruption is inevitable in China's pharmaceutical industry. www.c hina-briefing.com/news/2013/07/25/why-corruption-is-inevitable-in-chinas-pharmaceutical -industry.html (Zugriff vom 19.12.2013).

CNNMoney 2013. »Home prices spike 15\% in key Chinese cities «, in CNNMoney vom 18. September 2013.

Cox, Simon 2011. "Keynes v Hayek in China, China spent 2011 worrying about others' debt problems. In 2012 it will face one of its own ", in The Economist vom 17. November 2011.

Cox, Simon 2012. "Hayek on the standing committee. Who is winning the battle of economic ideas in China?", in The Economist vom 15. September 2012.

Das, Satyajit 2010. Traders, guns \& money - knowns and unknowns in the dazzling world of derivatives. New Jersey: Prentice Hall - Pearsoned Ltd.

Der Spiegel 2012. »New Ordos: Chinas gigantische Geisterstadt «, in Spiegel online, 17. März 2012. www.spiegel.de/reise/aktuell/new-ordos-ist-eine-geisterstadt-in-china-in-der-innerenmongolei-a-821998.html (Zugriff vom 19.12.2013).

Der Spiegel 2013. »Martin Winterkorn: Chinas Machthaber macht Volkswagen-Chef zum TopBerater ", in Spiegel online, 5. Juni 2013. www.spiegel.de/wirtschaft/unternehmen/vw-chefwinterkorn-beraet-chinas-ministerpraesidenten-a-904013.html (Zugriff vom 19.12.2013).

Die Presse 2008. » Russland und China legen Grenzstreit endgültig bei«, in Die Presse vom 21. Juli 2008. 
Die Welt 2013 a. »Russland und China kaufen weltweit Goldbestände auf «, in Die Welt vom 11. Februar 2013.

Die Welt 2013 b. »Microsoft übernimmt Handy-Geschäft von Nokia«, in Die Welt vom 9. September 2013.

Dohms, Heinz-Roger 2013. »G 20 und Schattenbanken - Das 70-Billionen-Dollar-Problem «, in DIE ZEIT vom 5. September 2013.

Eckholm, Erik 2000. "Big smuggling ring with a wide reach scandalizes China «, in The New York Times vom 22. Januar 2000, S. A1.

Eichengreen, Barry 2011. Exorbitant privilege - the rise and fall of the Dollar and the future of the International Monetary System. Oxford: Oxford University Press.

Eichengreen, Barry; Park, Donghyun; Shin, Kwanho 2013. Growth slowdowns redux: new evidence on the middle-income trap. NBER-Working Paper 18673. Cambridge: National Bureau of Economic Research.

Erber, Georg 2013 a. »Wirtschaftsbeziehungen zwischen China und der EU, der Eurozone und Deutschland ", in Weiterentwicklung der Keynes'schen Theorie und empirische Analysen, hrsg. v. Kromphart, Jürgen, S. 223-262. Marburg: Metropolis-Verlag.

Erber, Georg 2013 b. »Deutsch-chinesische Wirtschaftsbeziehungen: Chancen und Risiken für Deutschland «, in DIW-Wochenbericht 80, 41, S 9-15.

Euler-Hermes 2013. The reindustrialization of the United States. Economic Outlook, Special Report No. 1187.

Evolve 2012. The journey from offsourcing to onshoring (White Paper). Mountain View: Evolve Manufacturing Technologies Inc.

FAZ 2010. "Welthandel: Ostasien schafft größte Freihandelszone der Welt", in Frankfurter Allgemeine Zeitung vom 3. Januar 2010.

FAZ 2013 a. "Euro-Währungsreserven - Weiter die Nummer zwei «, in Frankfurter Allgemeine Zeitung vom 11. Januar 2013.

FAZ 2013 b. »Verhandlungen abgeschlossen. China und die Schweiz schließen Freihandelsabkommen ", in Frankfurter Allgemeine Zeitung vom 6. Juli 2013

FAZ 2013 c. »Gemeinsamer Devisen-Fonds: Schwellenländer treffen Vorsorge gegen Kursstürze", in Frankfurter Allgemeine Zeitung vom 6. September 2013.

Fisman, Raymond; Migual, Edward 2008. Economic gangsters: corruption, violence, and the poverty of nations. Princeton: Princeton University Press.

Freeman, Richard B. 1995. "Are our wages set in Beijing? ", in Journal of Economic Perspectives 9, 3, S. 15-32.

Fung, Anita 2013. »RMB on a reserve currency roll«, in China Daily vom 17. April 2013.

Geinitz, Christian 2013. "Ni hao aus Peking: China bekommt ein neues Konjunkturpaket ", in Frankfurter Allgemeine Zeitung vom 25. Juli 2013.

Giesen, Christoph 2013. »Chinesischer Staatsfonds CIC: Investment streng nach Einkaufszettel «, in Süddeutsche Zeitung vom 9. Januar 2013.

Giles, Judith A.; Williams, Cara L. 1999. Export-led growth: a survey of the empirical literature and some non-causality results econometrics. EWP Working Paper 9901. Victoria, Canada: University of Victoria.

Handelsblatt 2008. »China legt massives Konjunkturprogramm auf «, in Handelsblatt vom 8. November 2008.

Handelsblatt 2013 a. »Rat der Topmanager. Winterkorn berät Chinas Ministerpräsidenten «, in Handelsblatt vom 5. Juni 2013. www.handelsblatt.com/unternehmen/industrie/rat-der-top manager-winterkorn-beraet-chinas-ministerpraesidenten/8305150.html (Zugriff vom 19.12.2013).

Handelsblatt 2013 b. »BMWs riskante China-Strategie«, in Handelsblatt vom 24. Mai 2013.

Handelsblatt 2013 c. "Kuschelkurs mit dem Staatsbetrieb. Daimler baut in China erstmals Motoren - und teilt sein Ingenieurswissen ", in Handelsblatt vom 19. November 2013, S. 19.

Handelsblatt 2013 d. »Chinas ICE ist begehrt. Thailand will Hochgeschwindigkeitszüge kaufen «, in Handelsblatt vom 19. November 2013, S. 23.

Hayek, Friedrich A. von 1943. Der Weg zur Knechtschaft. Erlenbach-Zürich: Eugen Rentsch. Hayek, Friedrich A. von 1973. »Die Anmaßung von Wissen «, in Ordo 26, S. 12-21.

Hille, Kathrin 2013. »Crackdown on shadow financing caused cash crunch, says China «, in Financial Times vom 23. Juni 2013.

Leviathan, 42. Jg., 1/2014 
Huang, Yasheng 2008. Capitalism with Chinese characteristics. New York: Cambridge University Press.

Hvistendahl, Mara 2012. Unnatural selection: choosing boys over girls, and the consequences of a world full of men. Philadelphia: Perseus Book.

Isaksson, Anders 2010. Public capital, infrastructure and industrial development, research and statistics branch. WP 15/2009. Wien: United Nations Industrial Development Organization.

IWF 2013. Fiscal monitor - fiscal adjustment in an uncertain world. Washington: Internationaler Währungsfond.

Kennedy, Paul 2010. The rise and fall of the great powers - economic change and military conflict from 1500 until 2000. New York: Random House.

Keynes, John Maynard 1991 [1925]. "Am I a liberal? ", in John Maynard Keynes: Essays in persuasion, S. 312-322. New York: W. W. Norton \& Company.

Kinkel, Steffen 2012. »Trends in production relocation and backshoring activities: changing patterns in the course of the global economic crisis ", in International Journal of Operations \& Production Management 32, 6, S. 696-720.

Kissinger, Henry 2011. On China. New York: Penguin Book.

Kolonk, Petra 2013. »Die sieben Sünden des Kommunisten «, in Frankfurter Allgemeine Zeitung vom 29. Juni 2013.

Melo, Jaime de; Robinson, Sherman 1990. Productivity and externalities models of export-led growth. The World Bank Working Papers 387. Washington: The World Bank.

Mill, John Stuart 2003 [1859]. On liberty and other writings, hrsg. v. Collini, Stefan. Cambridge: Cambridge University Press.

Munich Innovation Group 2013. China investiert - Patentportfolios und Investitionsstrategien chinesischer Firmen. München: Munich Innovation Group in Kooperation mit der Technischen Universität München.

Murdoch, Scott; Chambers, Matt 2013. »Chinese currency agreement set to deliver a welcome boost for non-mining exporters", in The Australian vom 9. April 2013.

Nye, Joseph S. 1990. "Soft power ", in Foreign Policy 80, 3, S. 153-171.

Pettis, Michael 2013. The great rebalancing: trade, conflict, and the perilous road abead for the world economy. Princeton: Princeton University Press.

Pilling, David 2013. »Beijing's internet crackdown may be hard to sustain ", in Financial Times vom 19. September 2013.

Pilny, Karl H. 2005. Das asiatische Jahrhundert: China und Japan auf dem Weg zur neuen Weltmacht. Frankfurt a. M.: Campus.

Pommeranz, Kenneth 2000. Great divergence: China, Europe and the making of the modern world economy. Princeton economic history of the western world. Princeton: Princeton University Press.

PriceWaterhouseCoopers 2013. Standing out from competition - transfer pricing in China. www.pwc-prc.com/webmedia/doc/635056157574901914_tp_in_china_brochure.pdf (Zugriff vom 19.12.2013).

Rabinovitch, Simon 2013. »China's rising property prices fuelled by fear of missing out «, in Financial Times vom 19. September 2013.

Reuters 2012. China's Hu says graft threatens state; party must stay in charge. Meldung vom 8. November 2012.

Reuters 2013. »China stemmt sich gegen Wirtschaftsabschwächung «, in Die Welt vom 25. Juli 2013.

Reinhart, Carmen M.; Rogoff, Kenneth S. 2010. Growth in a time of debt. NBER Working Paper No. 15639. Cambridge: National Bureau of Economic Research.

Rimmele, Markus 2013. "Freihandelszone in Schanghai eröffnet - Tabubruch im Testbetrieb «, in tagesschau.de vom 29. September 2013. www.tagesschau.de/wirtschaft/schanghai-freihandelszone110.html (Zugriff vom 19.12.2013).

Rheinpfalz 2013. »Chinas Hochgeschwindigkeitszug ist ein Exportschlager «, in Reinpfalz vom 2. Dezember 2013.

Roberts, Dexter 2013 a. "China's shadow banking sector tops $\$ 5.8$ trillion, report says ", in Businessweek vom 8. Mai 2013. 
Roberts, Dexter 2013 b. "China profit rise masks state enterprise weakness ", in Businessweek vom 27. Juni 2013.

Saxenian, Anna Lee 2006. The new argonauts. Cambridge: Harvard University Press.

Schularick, Moritz; Taylor, Alan M. 2009. Credit booms gone bust: monetary policy, leverage cycles and financial crises, 1870-2008. NBER Working Paper No. 15512. Cambridge: National Bureau of Economic Research.

Siemons, Mark 2013. »Über sieben Grenzen müsst ihr gehen «, in Frankfurter Allgemeine Zeitung vom 27. September 2013.

Süddeutsche Zeitung 2013. »Chinesischer Ex-Minister zu Todesstrafe auf Bewährung verurteilt ", in Süddeutsche Zeitung vom 8. Juli 2013.

The Economist 2011. »Government's role in industry - the long arm of the state. Special report ", in The Economist vom 23. Juni 2011.

Verick, Sher; Islam, Iyanatul 2010. The great recession of 2008-2009: causes, consequences and policy responses. IZA-Working Paper, DP No. 4934. Bonn: IZA.

Volcker, Paul; Gyohten, Toyoo 1992. Changing fortunes. The world's money and the threat to American leadership. New York: Times Books/Random House.

Walter, Carl E.; Howie, Fraser J. T. 2012. Red capitalism - the fragile financial foundation of China's extraordinary rise. Singapore: Wiley \& Sons Asia.

Wapshot, Nicholas 2011. Keynes - Hayek - the clash that defined modern economics. New York, London: Norton \& Company.

Weltbank 2013. China 2030 - building a modern, harmonious, and creative society. Washington: Weltbank in Kooperation mit dem Development Research Center of the State Council, the People's Republic of China.

Weihua, Chen 2010. »Multinationals under scrutiny for corruption«, in China Daily vom 8. September 2010.

Wicksell, Knut 2005 [1898]. Geldzins und Güterpreise. München: FinanzBuch Verlag.

Wildau, Gabriel 2013. "Insight: Zombie borrowers haunt China's shadow banks", in Reuters vom 23. August 2013.

Xinhua 2004. "China to bailout two more state banks «, in China Daily vom 12. März 2004. www.chinadaily.com.cn/english/doc/2004-12/03/content_397161.htm (Zugriff vom 19.12.2013).

Xinhua 2011. »China confiscates 70 million fake invoices «, in China Daily vom 13. Juli 2011.

Xinhua 2012. »Chinas Hochgeschwindigkeitsnetz wächst rapide", in German.China.org.CN vom 17. Februar 2012. http://german.china.org.cn/business/txt/2012-02/17/content_2466 5737.htm (Zugriff vom 19.12.2013).

Yajing, Zhao; Jie, Woo Jun 2013. »China has need of well-run state-owned enterprises «, in South China Morning Post vom 28. August 2013.

Yanqi, Tong; Shaohua, Lei 2010. »Large-scale mass incident in China «, in East Asian Policy 2, 2, S. 23-33.

Yanqi, Tong; Shaohua, Lei 2011. "Wen pledges to curb graft, income inequality as police head off protests ", in Bloomberg News vom 28. Februar 2011. www.bloomberg.com/news/ 2011-02-27/china-police-blanket-planned-jasmine-protest-sites-in-beijing-shanghai.html (Zugriff vom 19.12.2013).

Zinn, Karl Georg 2013. Keynes contra Keynes? Zur Rezeption der Wachstumsskepsis des älteren Keynes. Vortrag auf der Tagung der Keynes-Gesellschaft am 18./19. Februar 2013 in Berlin.

Zusammenfassung: China wurde während der zurückliegenden Dekade aufgrund seines hohen Wirtschaftswachstums die zweitgrößte Volkswirtschaft nach den USA. Ob China die USA überholen wird, hängt jedoch entscheidend davon $a b$, wie China den komplizierten Anpassungsprozess schaffen kann. Vom bisherigen Wachstumsmodell, basierend auf billigen Arbeitskräften, exportorientiertem Wachstum und einem Bauboom, muss China zu einem Wachstum auf der Grundlage von eigenständigen Innovationen und hochqualifizierten Arbeitskräften gelangen. Dabei muss es stärker als bisher auch die Bedürfnisse des chinesischen Binnenmarktes befriedigen, da die übrige Welt immer weniger bereit, ist hohe Handelsbilanzüberschüsse hinzunehmen. Ansonsten droht es in der middle-income trap wie zuvor

Leviathan, 42. Jg., 1/2014 
andere rasch wachsende Länder steckenzubleiben. China ist durch die Integration in die Weltwirtschaft verletzlicher geworden gegenüber externen Schocks, die in der Krise von 2008 bisher nur mit keynesianischer Nachfragesteuerung und entsprechender Staatsverschuldung abgewendet werden konnten. Der weiterhin hohe Anteil an Staatsunternehmen hat maßgeblich dazu beigetragen, diese für falsche Prestigewettkämpfe und Korruption von Regierungsvertretern und Parteifunktionären anstelle marktwirtschaftlichen Wettbewerbs einzusetzen. Dies hat auch die Staatsführung in eine Legitimationskrise geführt, die gewachsene Ungleichheit bei Einkommen und Vermögen hat soziale Unruhen provoziert, und die Frage stellt sich, ob mehr Liberalisierungsschritte ein Innovationspotenzial der Privatwirtschaft freisetzen können. Das würde freilich zum Kontrollverlust durch Partei und Staat zu einem Zeitpunkt führen, an dem die chinesische Elite bemüht ist, die globale Rolle Chinas neu zu definieren: mit neuen militärstrategischen Ansprüchen, einer zunehmenden Präsenz chinesischer Unternehmen im Ausland und dem Versuch, den Renminbi zu einer Weltreservewährung zu machen. Daraus ergeben sich Widersprüche, da die knappen Ressourcen gleichzeitig für alle diese Ziele bereitgestellt werden müssen. Die Frage ist, ob es China gelingt, diese Zielkonflikte angemessen miteinander auszubalancieren.

Stichworte: China, Wirtschaftsentwicklung, Wirtschaftsreformen, Mittelstandsfalle

\section{China - country of endless opportunities?}

Summary: With its extraordinary high economic growth, China has become the second largest economy behind the US. Whether China will overtake the US depends on its ability to transform its past growth model based on cheap labor, export-led growth and high construction activities into a model based on innovation and high-skilled labor serving the domestic market more than it has until now. Greater efforts must be made to satisfy the needs of the Chinese domestic market, since the rest of the world is increasingly unwilling to accept it's high trade surpluses. Otherwise there is the danger that it will be caught in the middleincome trap which has infected other rapidly growing countries. China's integration into the global economy has made it more vulnerable to external shocks, which following the crisis in 2008 has been averted by Keynesian demand management and high public deficits. The large number of state-owned enterprises has opened the door for detrimental prestige competition and corruption among government officials, the Chinese regime tends to lose more and more legitimacy in the Chinese population. Inequality in China has already reached high levels which leads to rising social unrest. The question arises whether major liberalization steps will be able to generate previously unused innovative potentials. This would loosen control of the Chinese government and its Communist Party elite at a time when this elite is trying to define the Chinese role anew: With new military options, attempts are being made for a stronger foreign presence of Chinese corporations and to make the Renminbi a global reserve currency. All these activities create a trade-off to attain all these aims, since resources are scarce.

Keywords: China, economic development, economic reforms, middle-income trap

Autor

Dr. Georg Erber

Arbeitsbereich Märkte und Regulierung

Deutsches Institut für Wirtschaftsforschung

Mohrenstr. 58

10117 Berlin

georg.erber@hotmail.de 


\section{Appendix}

Chinesische Unternehmen unter den Fortune Global 500 im Jahr 2012

\begin{tabular}{|c|c|c|c|c|}
\hline in China & \multirow{3}{*}{ Unternehmen } & Global & \multirow{3}{*}{ City } & Umsatz \\
\hline \multirow{2}{*}{ Rankg】 } & & 500 & & \multirow{2}{*}{ (\$ millions) } \\
\hline & & Rang & & \\
\hline 1 & Sinopec Group & 5 & Beijing & 375,214 \\
\hline 2 & China National Petroleum & 6 & Beijing & 352,338 \\
\hline 3 & State Grid & 7 & Beijing & 259,142 \\
\hline 4 & Industrial \& Commercial Bank of China & 54 & Beijing & 109,040 \\
\hline 5 & China Construction Bank & 77 & Beijing & 89,648 \\
\hline 6 & China Mobile Communications & 81 & Beijing & 87,544 \\
\hline 7 & Agricultural Bank of China & 84 & Beijing & 84,803 \\
\hline 8 & Noble Group & 91 & Hong Kong & 80,732 \\
\hline 9 & Bank of China & 93 & Beijing & 80,230 \\
\hline 10 & China State Construction Engineering & 100 & Beijing & 76,024 \\
\hline 11 & China National Offshore Oil & 101 & Beijing & 75,514 \\
\hline 12 & China Railway Construction & 111 & Beijing & 71,443 \\
\hline 13 & China Railway Group & 112 & Beijing & 71,263 \\
\hline 14 & Sinochem Group & 113 & Beijing & 70,990 \\
\hline 15 & China Life Insurance & 129 & Beijing & 67,274 \\
\hline 16 & SAIC Motor & 130 & Shanghai & 67,255 \\
\hline 17 & Dongfeng Motor Group & 142 & Wuhan & 62,911 \\
\hline 18 & China Southern Power Grid & 152 & Guangzhou & 60,538 \\
\hline 19 & China FAW Group & 165 & Changchun & 57,003 \\
\hline 20 & China Minmetals & 169 & Beijing & 54,509 \\
\hline 21 & CITIC Group & 194 & Beijing & 49,339 \\
\hline 22 & Baosteel Group & 197 & Shanghai & 48,916 \\
\hline 23 & China North Industries Group & 205 & Beijing & 48,154 \\
\hline 24 & China Communications Construction & 216 & Beijing & 45,959 \\
\hline 25 & China Telecommunications & 221 & Beijing & 45,170 \\
\hline 26 & China Resources National & 233 & Hong Kong & 43,440 \\
\hline 27 & Shenhua Group & 234 & Beijing & 43,356 \\
\hline 28 & China South Industries Group & 238 & Beijing & 43,160 \\
\hline 29 & Ping An Insurance & 242 & Shenzhen & 42,110 \\
\hline 30 & China Huaneng Group & 246 & Beijing & 41,481 \\
\hline 31 & Aviation Industry Corp. of China & 250 & Beijing & 40,835 \\
\hline 32 & China Post Group & 258 & Beijing & 40,023 \\
\hline 33 & HeBei Iron \& Steel Group & 269 & Shijiazhuang & 38,722 \\
\hline 34 & Jardine Matheson & 275 & Hong Kong & 37,967 \\
\hline 35 & China Metallurgical Group & 280 & Beijing & 37,613 \\
\hline 36 & People's Insurance Co. of China & 292 & Beijing & 36,549 \\
\hline Quelle: F & rtune 500 Global 2012. & & & \\
\hline
\end{tabular}




\begin{tabular}{|c|c|c|c|c|}
\hline in China & \multirow{3}{*}{ Unternehmen } & Global & \multirow{3}{*}{ City } & Umsatz \\
\hline \multirow{2}{*}{ Rankg ${ }^{\top}$} & & 500 & & \multirow{2}{*}{ (\$ millions) } \\
\hline & & Rang & & \\
\hline 37 & Shougang Group & 295 & Beijing & 36,117 \\
\hline 38 & Aluminum Corp. of China & 298 & Beijing & 35,839 \\
\hline 39 & China National Aviation Fuel Group & 318 & Beijing & 34,352 \\
\hline 40 & Wuhan Iron \& Steel & 321 & Wuhan & 34,260 \\
\hline 41 & Bank of Communications & 326 & Shanghai & 33,872 \\
\hline 42 & Jizhong Energy Group & 330 & Xingtai & 33,661 \\
\hline 43 & China United Network Communications & 333 & Shanghai & 33,336 \\
\hline 44 & China Guodian & 341 & Beijing & 32,580 \\
\hline 45 & Jiangsu Shagang Group & 346 & Zhangjiagang & 32,097 \\
\hline 46 & China Railway Materials & 349 & Beijing & 31,991 \\
\hline 47 & Huawei Investment \& Holding & 351 & Shenzhen & 31,543 \\
\hline 48 & Hutchison Whampoa & 362 & Hong Kong & 30,023 \\
\hline 49 & China National Building Materials Group & 365 & Beijing & 30,022 \\
\hline 50 & Sinomach & 367 & Beijing & 29,846 \\
\hline 51 & China Datang & 369 & Beijing & 29,603 \\
\hline 52 & Lenovo Group & 370 & Beijing & 29,574 \\
\hline 53 & China Ocean Shipping & 384 & Beijing & 28,797 \\
\hline 54 & Power China & 390 & Beijing & 28,289 \\
\hline 55 & COFCO & 393 & Beijing & 28,190 \\
\hline 56 & Henan Coal \& Chemical & 397 & Zhengzhou & 27,919 \\
\hline 57 & ChemChina & 402 & Beijing & 27,707 \\
\hline 58 & Tewoo Group & 416 & Tianjin & 26,411 \\
\hline 59 & China Electronics & 425 & Beijing & 26,023 \\
\hline 60 & Zhejiang Materials Industry Group & 426 & Hangzhou & 25,833 \\
\hline 61 & China Huadian & 433 & Beijing & 25,270 \\
\hline 62 & China Shipbuilding Industry & 434 & Beijing & 25,145 \\
\hline 63 & Shandong Weiqiao Pioneering Group & 440 & Shandong & 24,906 \\
\hline 64 & Shanxi Coal Transportation \& Sales Group & 447 & Taiyuan & 24,533 \\
\hline 65 & China Pacific Insurance (Group) & 450 & Shanghai & 24,429 \\
\hline 66 & China Power Investment & 451 & Beijing & 24,400 \\
\hline 67 & Shandong Energy Group & 460 & Jinan City & 24,131 \\
\hline 68 & Ansteel Group & 462 & Anshan & 24,089 \\
\hline 69 & Zhejiang Geely Holding Group & 475 & Hangzhou & 23,356 \\
\hline 70 & Greenland Holding Group & 483 & Shanghai & 22,873 \\
\hline 71 & Xinxing Cathay International Group & 484 & Beijing & 22,832 \\
\hline 72 & Kailuan Group & 490 & Tangshan & 22,519 \\
\hline 73 & China Merchants Bank & 498 & Shenzhen & 22,094 \\
\hline Quelle: Fc & une 500 Global 2012. & & & \\
\hline & & & & \\
\hline
\end{tabular}

